\begin{tabular}{|c|l|}
\hline Title & Should income inequality be prai sed? Multiple public goods provision, income distribution, and social welfare \\
\hline Author(s) & Itaya, Jun-ichi; Mizushima, A tsue \\
\hline Citation & Discussion Paper, Series A, 298, 1-37 \\
\hline Issue Date & 2016-01-26 \\
\hline Doc URL & http:/hdl.handle.net/2115/60614 \\
\hline Type & bulletin (article) \\
\hline File Information & DPA 298.pdf \\
\hline
\end{tabular}

Instructions for use 
Discussion Paper, Series A, No.2016-298

Should income inequality be praised? Multiple public goods provision, income distribution, and social welfare

Jun-ichi Itaya

Atsue Mizushima

January.2016

Graduate School of Economics \& Business Administration

Hokkaido University

Kita 9 Nishi 7, Kita-Ku, Sapporo 060-0809, JAPAN 


\title{
Should income inequality be praised? Multiple public goods provision, income distribution, and social welfare*
}

\author{
Jun-ichi Itaya ${ }^{\dagger} \quad$ Atsue Mizushima
}

January 26, 2016

\begin{abstract}
We investigate how income inequality affects social welfare in a model of voluntary contributions to multiple pure public goods. Itaya, de Meza, and Myles (1997) show that the maximization of social welfare precludes income equality in a single pure public good model. In contrast, we show that the result of Itaya et al. may not be valid in a case of multiple voluntarily supplied public goods; specifically, we show that not only an income inequality-raising redistribution policy but also an income-equalizing one may raise social welfare. We also show that if altruistically motivated voluntary transfers are allowed, an inequality-raising redistribution policy is no longer effective and leaves social welfare unchanged.
\end{abstract}

JEL classification: D31, D63, D64, H23, H31

Keywords: Public goods, Inequality, Social welfare, Voluntary provision, Income distribution

${ }^{*}$ We would like to thank Professors Tatsuyoshi Saijo, Youngsub Chun, Akihisa Shibata, Koichi Futagami, and Hisahiro Naito for their useful comments and helpful suggestions. We also thank the Seoul National University seminar participants and the participants at the 15th Annual Conference of Public Economic Theory at the University of Washington, Seattle, the 70th Annual Congress of the International Institute of Public Finance, Lugano, Switzerland, and the 2015 Autumn Meeting of the Japanese Economic Association at Sophia University, Tokyo. This research was supported by Grant-in-Aid for Scientific Research from the Japan Society for the Promotion of Science (\#26380355). All errors remaining in the paper are our own responsibility.

${ }^{\dagger}$ Corresponding author: Graduate School of Economics, Hokkaido University, N9W7 Kita-ku, Sapporo, Hokkaido 060-0809, Japan; E-mail:itaya@econ.hokudai.ac.jp

${ }^{\ddagger}$ Faculty of Economics, Otaru University of Commerce, 3-5-21 Midori, Otaru, Hokkaido 047-8501, Japan; E-mail:mizushima@res.otaru-uc.ac.jp 


\section{Introduction}

This paper studies how income inequality affects social welfare in a voluntary contributions model of multiple pure public goods. In a voluntary contributions model with a single public good, Bergstrom, Blume, and Varian (1986) show that every income redistribution among the contributors will not affect the equilibrium levels of the consumption of private and public goods provisions (which is in line with the so-called Warr's (1983) neutrality theorem) and hence does not alter the individuals' utility levels, but a transfer of income from noncontributors to contributors increases the total supply of a public good. Furthermore, Itaya, de Meza, and Myles (1997) show that the maximization of social welfare precludes income equality. More precisely, they show that a transfer of income from a marginal contributorthat is, one who is indifferent between contribution and non-contribution - to contributors is desirable from the perspective of social welfare; in short, income inequality-raising transfers rather than income-equalizing ones is socially praiseworthy.

However, the theoretical prediction of Itaya et al. (1997) seems to be inconsistent with casual observations on actual economies as well as the widely accepted belief that public policies such as those on tax and transfers are to reduce income inequality. For instance, for the OECD countries over 2012 (see OECD, 2012), the Gini coefficients on pre-taxes and transfers basis for the total population are ranged between 0.34 and 0.53 , with South Korea showing the lowest and Italy the highest, whereas the Gini coefficients on after-taxes and transfers basis for the total population are ranged between 0.25 and 0.48 , with Denmark showing the lowest and Mexico the highest. For the United States, the country with the largest population among the OECD countries, the pre-tax Gini index was 0.49 and the aftertax Gini index 0.38 in 2012. Taken together, the OECD average for total population in the OECD countries was 0.46 for the pre-tax income Gini index and 0.31 for the after-tax income Gini Index. Overall, it seems that the Gini indices on after-taxes and transfers basis in the respective OECD countries become significantly smaller, despite large variations across the OECD countries, implying that all OECD countries certainly show redistributive effects.

Moreover, a large number of persons including economists and politicians agree with the 
view that income redistribution - generally understood as a mix of progressive taxation, minimum wage law, tax credits, and cash transfers or payment-in-kind to lower-income groups in order to diminish the earned income inequalities between social strata - is a desirable and justifiable social goal on grounds of fairness or equity in democratic, humanistic societies. Equity is the appropriate baseline norm for end-results equity or distributive justice in most countries. Thus, most public economists agree that allowing inequalities to exist can be justified on only the ground of marginal cost of "Okun's leaky bucket," representing the efficiency costs of taxing and transferring. The apparent discrepancy between the proceeding observations as well as prevailing views and the above claim made by Itaya et al (1997) not only casts serious doubts on the validity of their conclusion, but also induces us to question the appropriateness of the basic assumptions of their model. In particular, we re-examine the effect of the income redistribution policy on social welfare in a private provision model of multiple pure public goods rather than the standard model of a single pure public good. The motivation for this extension and the main results of this study are summarized as follows.

First, we easily find numerous examples from day-to-day life where individuals or groups contribute simultaneously to more than one pure public good. Individuals voluntarily contribute to several charitable trusts, non-profit organizations (NPOs), or Parent-Teacher Associations. Furthermore, family members make simultaneously voluntary contributions to multiple household public goods such as caring for the old, children, and sick family members, housework, and gardening. ${ }^{1}$ Moreover, at the macro level, national governments simultaneously choose to allocate budgets for the provision of several national public goods such as public health care and infrastructure as well as several international public goods such as military alliances and foreign aid.

Second, although ample examples exist in reality, the literature presents only a limited number of explicit analyses of voluntary contribution models with many pure public goods, such as Kemp (1984), Bergstrom, Blume, and Varian (1986), Cornes and Schweinberger (1996), and Cornes and Itaya (2010). Among these, Cornes and Itaya (2010) is the work most closely related to the present paper, examining a simple model of voluntary public goods provision

\footnotetext{
${ }^{1}$ Browning et al. (2014) provide more examples on the economics of family.
} 
that allows for two or more public goods and showing that the equilibrium of voluntary contributions typically generates not only a very low level of public goods provision but also the wrong mix of public goods such that variations in the existing combination of several public goods could lead to Pareto-improving allocations. Unfortunately, none of the studies has seriously investigated the effect of income distribution or a redistribution policy on social welfare. The exception is Gohosh et al. (2007), who show that for large income inequalities among individuals possessing identical preferences, bundling of several public goods leads to higher social welfare, whereas for small income inequality among individuals with heterogeneous preferences, bundling may decrease social welfare in the model of multiple voluntarily supplied public goods. However, Gohosh et al. focus only on the effects of bundling of multiple public goods on social welfare rather than the welfare effect of a redistribution policy, which is of more substantive interest in the literature on private provision of public goods, and which is the task we take up in this study.

Third, several papers have explored the possibility of a Pareto-improving income distribution using a single voluntarily provided public good model. Cornes and Sandler (2000) show that when the number of contributors is larger than a certain number, redistribution from the poor to the rich can lead to Pareto improvement. More recently, Dasgupta (2009) demonstrated that the claim made by Itaya et al. (1997) breaks down when the public good is impure. Mukherjee and Sanyal (2011) point out the possibility of Pareto improvement in an economy consisting of two persons when the non-contributing poor tolerate increasing income inequality. The analysis of voluntary provision models with multiple public goods is out of the scope of their studies. In contrast, we consider a two-individual Nash game of voluntary provision of multiple public goods and investigate how the egalitarian (or, more generally, symmetric) social welfare function varies in response to different income distributions in a society. We first conduct this analysis under general quasi-concave utility functions and then under identical and non-identical Cobb-Douglas utility functions with and without altruistic preferences toward others. Our noteworthy finding is that the result of Itaya et al. would be valid even in the presence of multiple voluntarily supplied public goods; that is, an inequalityraising redistribution policy raises social welfare when the distribution of income is extremely 
unequal, whereas an income-equalizing redistribution policy may also raise social welfare when the income of different individuals do not differ very much.

Fourth, we move beyond the egoistic assumption that contributors care only for their own well-being and thus allow for altruism toward others. For instance, altruistically motivated family members care about one another and thus make simultaneous private income transfers to other family members and positive contributions to household public goods. If one individual were to have most of the income, that individual would voluntarily make a positive transfer of income to poorer individuals and at the same time be the sole contributor to several public goods. We find that in this case, an inequality-raising redistribution policy will be neutralized by voluntary income transfers, ultimately leaving social welfare unaffected. In other words, an inequality-raising redistribution policy is no longer effective as long as altruistic motivations work.

This paper is organized as follows. Section 2 presents a basic model with general quasiconcave utility functions but without altruistic preferences, characterizes equilibrium outcomes, and conducts welfare analysis of income inequality-raising and income-equalizing redistribution policies. Section 3 considers a model with quasi-concave utility functions and altruistic preferences. Section 4 investigates the effects on social welfare under identical CobbDouglas utility functions with and without altruistic motives respectively. Section 5 conducts the same analysis under non-identical Cobb-Douglas utility functions with and without altruistic preferences respectively. Section 6 concludes the paper with a discussion of some possible extensions of the model.

\section{The Model}

\subsection{Framework: Egoistic Preferences}

We consider private provision of multiple public goods as in Bergstrom, Blume, and Varian (1986) and Cornes and Itaya (2010). Two individuals are indexed by $i=1,2$, each dividing her income between private consumption, $c_{i}$, and contributions toward two distinct pure public goods, $G$ and $H$, respectively denoted by $g_{i}$ and $h_{i}$. The preferences of individual $i$ are given 
by $u^{i}\left(x_{i}, G, H\right)$. Since, as in the literature, individuals are assumed to make contributions $g_{i}$ and $h_{i}$ simultaneously and noncooperatively, we use Nash equilibrium as our solution concept. Individual $i$ 's budget constraint is expressed as

$$
x_{i}+g_{i}+h_{i}=m_{i}
$$

where $m_{i}$ is the exogenously given income of individual $i$ and the relative price (unit costs of production) of public goods $G$ and $H$ relative to the (numeraire) private good is normalized to 1 . Furthermore, we make the following assumption:

Assumption 1 The utility function $u^{i}\left(x_{i}, G, H\right)$ of individual $i(i=1,2)$ is $(i)$ strictly quasiconcave, strictly increasing, and twice continuously differential with respect to each argument on $R_{++}^{3},(i i)$ it satisfies the Inada conditions $\lim _{G \rightarrow 0}\left(\partial u_{i}\left(x_{i}, G, H\right) / \partial G\right)=\infty$, similarly for $H$ and $x_{i},(i i i)$ the bordered Hessian of $u^{i}\left(x_{i}, G, H\right)$ is non-singular on $R_{++}^{3}$, and $(i v) x_{i}, G$, and $H$ are all normal goods for each individual.

The above assumption encompasses a wide variety of utility functions used in the literature and all our qualitative results hold for every functional form satisfying it.

Individual $i$ maximizes $u^{i}\left(x_{i}, G, H\right)$ by its choice of $x_{i}, g_{i}$, and $h_{i}$ subject to her budget constraint (1) and nonnegativity constraints $x_{i} \geq 0, g_{i} \geq 0$, and $h_{i} \geq 0$, given the contributions $G_{-i}$ and $H_{-i}$ of the others and the Nash conjecture that they will be unaffected by their own choices, where, as usual, $G_{-i}$ and $H_{-i}$ denote the total contribution by all individuals different from $i$ respectively. Thus, Nash equilibrium of the corresponding contribution game played by the two individuals is defined as follows:

Definition 1 Nash equilibrium in this model is a collection of strategies $\left\{\left(x_{i}, g_{i}, h_{i}\right) \in R_{+}^{3} \mid i=1,2\right\}$ such that $\left(x_{i}, g_{i}, h_{i}\right)$ is a solution to the following problem for $i=1,2$ :

$$
\begin{gathered}
\max _{x_{i}, g_{i}, h_{i}} u^{i}\left(m_{i}-g_{i}-h_{i}, g_{i}+G_{-i}, h_{i}+H_{-i}\right) \\
\text { s.t. } \quad x_{i} \geq 0, g_{i} \geq 0, h_{i} \geq 0 .
\end{gathered}
$$


The Kuhn-Tucker conditions for utility maximization of individuals $i=1,2$ defined by

Definition 1 are given $b y^{2}$

$$
\begin{gathered}
u_{G}^{i}\left(m_{i}-g_{i}-h_{i}, g_{i}+G_{-i}, h_{i}+H_{-i}\right)-u_{x}^{i}\left(m_{i}-g_{i}-h_{i}, g_{i}+G_{-i}, h_{i}+H_{-i}\right) \\
\leq 0 \text { for } g_{i} \geq 0, \\
u_{H}^{i}\left(m_{i}-g_{i}-h_{i}, g_{i}+G_{-i}, h_{i}+H_{-i}\right)-u_{x}^{i}\left(m_{i}-g_{i}-h_{i}, g_{i}+G_{-i}, h_{i}+H_{-i}\right) \\
\leq 0 \text { for } h_{i} \geq 0 .
\end{gathered}
$$

First, note that from Assumption $1(i)$, zero supply of $G$ or $H$ is excluded so that neither of the cases $g_{1}=g_{2}=0$ and $h_{1}=h_{2}=0$ is realized in equilibrium. Moreover, from Proposition 2 of Cornes and Itaya (2010), it is almost surely impossible to realize a case where everyone contributes to every public good except for identical preferences. ${ }^{3}$ These arguments imply eight possibilities associated with (2) and (3), which we need to consider:

Case $1: g_{1}=0, h_{1}>0, g_{2}>0$ and $h_{2}>0$,

Case $2: g_{1}>0, h_{1}=0, g_{2}>0$ and $h_{2}>0$,

Case $3: g_{1}>0, h_{1}>0, g_{2}=0$ and $h_{2}>0$,

Case $4: g_{1}>0, h_{1}>0, g_{2}>0$ and $h_{2}=0$,

Case $5: g_{1}=0, h_{1}=0, g_{2}>0$ and $h_{2}>0$

Case $6: g_{1}>0, h_{1}>0, g_{2}=0$ and $h_{2}=0$,

${ }^{2}$ For expositional clarity, we focus on interior solutions by ignoring the following situations:

$$
g_{i}=0 \text { when } u_{G}^{i}(.)=u_{x}^{i}(.) \text {, or } / \text { and } h_{i}=0 \text { when } u_{H}^{i}(.)=u_{x}^{i}(.) \text {. }
$$

Nevertheless, one or both of the above conditions should hold at the boundary point between adjacent cases.

${ }^{3}$ From Proposition 2 of Cornes and Itaya (2010), at least one of the first-order conditions should hold at inequality at a Nash equilibrium. Thus, at the Nash equilibrium of the multiple pure public goods model, there always exists at least one individual who is a non-contributor to one public good. The absence of an interior Nash equilibrium in contribution space is mainly due to a liner technology for supplying a public good. Their proposition further implies an exceptional case of identical preferences in which every individual can simultaneously contribute to all public goods at a Nash equilibrium. In this case, although the equilibrium levels of the respective public goods may be uniquely determined, individual contributions are indeterminate and so individuals face a coordination problem with respect to their individual contribution levels. 
Case $7: g_{1}>0, h_{1}=0, g_{2}=0$ and $h_{2}>0$,

Case $8: g_{1}=0, h_{1}>0, g_{2}>0$ and $h_{2}=0$.

\subsection{Effect of Income Inequality on Social Welfare}

To start with, we use the following theorem of Cornes and Itaya (2010) that not only generalizes the neutrality theorem of Warr (1983) to the case of multiple public goods, but also shows that in such a case, a "small" change in distribution of income, with the total level of income remaining unchanged, does not affect the allocation of resources: ${ }^{4}$

Proposition 1 (Proposition 4 of Cornes and Itaya, 2010) An income redistribution restricted to a set of linked individuals and that maintains the links between them has no effect on the original equilibrium allocation.

In the above theorem, the term "linked individuals" is defined as follows:

Definition 2 (Definition 3 of Cornes and Itaya, 2010) Individuals $h$ and $h+k$ are linked at an equilibrium if there is a set of public goods (labeled $G_{1}, G_{2}, \ldots, G_{k}$ ) and a set of individuals (labeled $h, h+1, \ldots, h+k)$ such that, at that equilibrium,

- Individual $h$ shares an active interest with individual $h+1$ in public good $G_{1}$,

- Individual $h+1$ shares an active interest with individual $h+2$ in public good $G_{2}$,

- Individual $h+k-1$ shares an active interest with individual $h+k$ in public good $G_{k}$.

Thus, if individuals $h$ and $h+k$ are linked through a sequence of positive contributions to several public goods, so too are any two individuals belonging to the chain linking them. In light of Proposition 1, the neutrality theorem holds in a multiple-public goods provision model provided the individuals involved are linked to each other, whereby the level of social

\footnotetext{
${ }^{4}$ The assumption of a "small" redistribution of income means that the size of redistributing income never exceeds his or her initial income. This assumption is commonly used in the literature on private provision of public goods to ensure the neutrality property in terms of redistribution of income.
} 
welfare remains unchanged with respect to the redistribution of income or different profiles of income distribution.

We can now analyze how the inequality of income (or a redistribution policy of income) affects social welfare in Cases 1-8. Before doing this, we make the following assumption:

Assumption 2 Social welfare function $W\left(u^{1}, u^{2}\right)$ is increasing in each argument and symmetric in the two individuals' utilities (i.e., $\partial W / \partial u^{1}=\partial W / \partial u^{2}$ if $\left.u^{1}=u^{2}\right){ }^{5}$

Armed with these assumptions, we present the following:

Cases 1-4: From Proposition 1 as well as Definition 1, it turns out that the two individuals in these cases are linked through positive contributions to the public good $G$ or $H$ so that the neutrality theorem holds. Thus, Proposition 1 implies that their private consumption and the total provision of the two public goods remain invariant in terms of a "small" redistribution of income (holding the sum of income of the two individuals fixed). Thus, we can conclude that within the range of income distribution associated with Cases 1-4, the utility levels of both individuals also remain constant with respect to changes in each individual's income share, as does the level of social welfare.

Cases 5 and 6: Let us first consider Case 5. At this Nash equilibrium, the Kuhn-Tucker conditions (2) and (3) are reduced to

$$
\begin{aligned}
& u_{G}^{2}\left(m_{2}-g_{2}-h_{2}, g_{2}, h_{2}\right)-u_{x}^{2}\left(m_{2}-g_{2}-h_{2}, g_{2}, h_{2}\right)=0 \text { for } g_{2}>0, \\
& u_{H}^{2}\left(m_{2}-g_{2}-h_{2}, g_{2}, h_{2}\right)-u_{x}^{2}\left(m_{2}-g_{2}-h_{2}, g_{2}, h_{2}\right)=0 \text { for } h_{2}>0,
\end{aligned}
$$

together with $g_{1}=0$ and $h_{1}=0$. Solving (4) and (5) yields $g_{2}\left(m_{2}\right)>0$ and $h_{2}\left(m_{2}\right)>0$. From the normality assumption (i.e., Assumption 1), it turns out that $d g_{2} / d m_{2}>0$ and $d h_{2} / d m_{2}>0$. By substituting these functions and denoting the levels of income $m_{1}$ and $m_{2}$

\footnotetext{
${ }^{5}$ Since we use the utilitarian type of social welfare function later so that the social welfare function generates a social preference ordering that completely ranks the alternative states, we have to impose cardinal unit comparability on the utility function of each individual, in the sense that the transformed utilities are restricted to have a common value for $b$, and so $\hat{u}^{i} \equiv a^{i}+b u^{i}$ for all individuals $i$, where $a^{i}$ and $b$ are arbitrary constants.
} 
by $\rho$ and $1-\rho$, respectively, we can use the indirect utility functions of both individuals, such as $u^{1}\left(\rho, g_{2}(1-\rho), h_{2}(1-\rho)\right)$ and $u^{2}\left(1-\rho-g_{2}(1-\rho)-h_{2}(1-\rho), g_{2}(1-\rho), h_{2}(1-\rho)\right)$, because we focus on the effect of a "small" redistribution of income, given a fixed level of total income (normalized to 1 ).

Suppose first that the initial income distribution is given by $(\bar{\rho}, 1-\bar{\rho})$, where individual 2 (whom we call "the rich" contributes to both public goods, $G$ and $H$, whereas individual 1 (whom we call "the poor" ) contributes to public good $G$ only; that is, Case 2 occurs first. ${ }^{6}$ Now, consider a small lump-sum transfer from individual 1 to 2 (i.e., $d \rho<0$ ), which we call "an inequality-raising redistribution," in the sense that the income of a non-contributor (= the poor $)$ is redistributed to a contributor $(=$ the rich $)$, in what follows. The transfer is made until the point where the resulting income level $\rho$ makes individual 1 indifferent between contributing and not contributing to the public good $G$; the distribution of income at that point is denoted as $(\hat{\rho}, 1-\hat{\rho})$ (the point also corresponds to the boundary point between Case 2 and Case 5). Since, from the proceeding argument, an infinitesimal redistribution of income does not affect social welfare as long as both individuals contribute at least one public good each to the supply of the same public good, and since the social welfare function continuously changes, the social welfare evaluated at $(\hat{\rho}, 1-\hat{\rho})$ should be equal to that at the initial distribution of income $(\bar{\rho}, 1-\bar{\rho})$; that is,

$$
\begin{gathered}
W\left(u^{1}\left(\bar{\rho}-g_{1}, g_{1}+g_{2}, h_{2}\right), u^{2}\left(1-\bar{\rho}-g_{2}-h_{2}, g_{1}+g_{2}, h_{2}\right)\right)= \\
W\left(u^{1}\left(\hat{\rho}, g_{2}, h_{2}\right), u^{2}\left(1-\hat{\rho}-g_{2}-h_{2}, g_{2}, h_{2}\right)\right)
\end{gathered}
$$

\footnotetext{
${ }^{6}$ As we move from individual 2, who has a larger income, to individual 1, who has a smaller income (holding total income constant), the number of public goods individual 1 contributes to will generally rise and the number of public goods individual 2 contributes to will generally fall, and, consequently, the rich generally contribute to a larger number of public goods than the poor do.
} 
By taking the left-hand side derivative of the right-hand side of (6) with respect to $\rho$, evaluated at the income distribution $(\hat{\rho}, 1-\hat{\rho})$, we can obtain the effect on social welfare $\mathrm{as}^{7}$

$$
\begin{gathered}
\left.\frac{d W}{d \rho}\right|_{\rho \rightarrow \hat{\rho}-}=W_{1} \frac{\partial u^{1}}{\partial x_{1}}-W_{1} \frac{\partial u^{1}}{\partial G} \frac{d g_{2}}{d(1-\rho)}-W_{1} \frac{\partial u^{1}}{\partial H} \frac{d h_{2}}{d(1-\rho)} \\
+W_{2} \frac{\partial u^{2}}{\partial x_{2}}\left[-1+\frac{d g_{2}}{d(1-\rho)}+\frac{d h_{2}}{d(1-\rho)}\right]-W_{2} \frac{\partial u^{2}}{\partial G} \frac{d g_{2}}{d(1-\rho)}-W_{2} \frac{\partial u^{2}}{\partial H} \frac{d h_{2}}{d(1-\rho)},
\end{gathered}
$$

where, recalling, $g_{2}=g_{2}(1-\rho)$ and $h_{2}=h_{2}(1-\rho)$. Here, we first consider the case of $i d e n t i c a l$ preferences across individuals, and from these identical preferences, we have the following lemma:

Lemma 1 (Theorem 2 in Itaya et al., 1997) Assume that all individuals have identical preferences. At the interior Nash equilibrium, all utility levels are equalized,

$$
V^{1}\left(m_{1}+m_{2}\right)=V^{2}\left(m_{1}+m_{2}\right)
$$

and the marginal utility of own and others' incomes are equalized,

$$
\frac{\partial V^{1}\left(m_{1}+m_{2}\right)}{\partial m_{1}}=\frac{\partial V^{2}\left(m_{1}+m_{2}\right)}{\partial m_{1}}
$$

where

$$
\begin{aligned}
V^{1}\left(m_{1}+m_{2}\right) & \equiv u^{1}\left(x_{1}\left(m_{1}+m_{2}\right), G\left(m_{1}+m_{2}\right), H\left(m_{1}+m_{2}\right)\right), \\
V^{2}\left(m_{1}+m_{2}\right) & \equiv u^{2}\left(x_{2}\left(m_{1}+m_{2}\right), G\left(m_{1}+m_{2}\right), H\left(m_{1}+m_{2}\right)\right) .
\end{aligned}
$$

By utilizing $\left(\partial u^{2} / \partial G\right) /\left(\partial u^{2} / \partial x_{2}\right)=1$ and $\left(\partial u^{2} / \partial H\right) /\left(\partial u^{2} / \partial x_{2}\right)=1$ (from (4) and (5)) and applying Lemma 1 along with the symmetric social welfare function, expression (7) can be simplified to

$$
\left.\frac{d W}{d \rho}\right|_{\rho \rightarrow \hat{\rho}-}=-W_{1} \frac{\partial u^{1}}{\partial G} \frac{d g_{2}}{d(1-\rho)}-W_{1} \frac{\partial u^{1}}{\partial H} \frac{d h_{2}}{d(1-\rho)}<0 .
$$

\footnotetext{
${ }^{7}$ We need to take the left-hand side derivative of the social welfare function $W$ at the switching point $(\hat{\rho}, 1-\hat{\rho})$, because this function is kinked at this point and thus its derivative does not exist.
} 
The above result implies that social welfare rises when income transfers are made from individual 1 (i.e., the poor) to individual 2 (i.e., the rich), that is, $d \rho<0$, because $d g_{2} / d(1-\rho)>0$ and $d h_{2} / d(1-\rho)>0$ (from the normality assumption).

Under non-identical preferences and from (4) and (5), on the other hand, we can rewrite (7) as follows:

$$
\left.\frac{d W}{d \rho}\right|_{\rho \rightarrow \hat{\rho}-}=W_{1} \frac{\partial u^{1}}{\partial x_{1}}-W_{1}\left[\frac{\partial u^{1}}{\partial G} \frac{d g_{2}}{d(1-\rho)}+\frac{\partial u^{1}}{\partial H} \frac{d h_{2}}{d(1-\rho)}\right]-W_{2} \frac{\partial u^{2}}{\partial x_{2}}
$$

where the second and third terms on the right-hand side of (8) are clearly negative whereas the first term is positive, implying that the sign of (8) is ambiguous. Nevertheless, we may say that if the socially weighted marginal utility of individual 2's (= the rich's) private consumption (i.e., $\left.W_{2}\left(\partial u^{2} / \partial x_{2}\right)\right)$ plus individual 1's (= the poor's) socially weighted marginal utility of the two public goods are greater than that of individual 1's private consumption (i.e., $\left.W_{1}\left(\partial u^{1} / \partial x_{1}\right)\right)$, then the sign of $(8)$ is negative, implying that the inequality-raising redistribution policy raises social welfare (recalling, $d \rho<0) .{ }^{8}$

The analysis of Case 6 follows the same lines as that of Case 5 .

Cases 7 and 8: First, let us consider Case 7. At this Nash equilibrium, the Kuhn-Tucker conditions (2) and (3) are reduced to

$$
\begin{gathered}
u_{G}^{1}\left(\rho-g_{1}, g_{1}, h_{2}\right)-u_{x}^{1}\left(\rho-g_{1}, g_{1}, h_{2}\right)=0 \text { for } g_{1}>0, \\
u_{H}^{2}\left(1-\rho-h_{2}, g_{1}, h_{2}\right)-u_{x}^{2}\left(1-\rho-h_{2}, g_{1}, h_{2}\right)=0 \text { for } h_{2}>0,
\end{gathered}
$$

with $g_{2}=0$ and $h_{1}=0$. By solving these conditions, we obtain $g_{1}(\rho, 1-\rho)>0$ and $h_{2}(1-\rho, \rho)>0$ (see Appendix A).

Let the initial distribution of income be given by $(\tilde{\rho}, 1-\tilde{\rho})$, where individual 2 contributes to both public goods whereas individual 1 contributes to public good $G$ only; that is, Case 2 occurs first. Consider the transfer of income from individual 2 (= the rich) to individual

\footnotetext{
${ }^{8}$ More specifically, we can show that under individuals' Cobb-Douglas utility functions and with some plausible parameter restriction (see Assumption 4), the level of social welfare unambiguously increases as a result of the inequality-raising redistribution policy; see Section 5.
} 
1 (= the poor) until the point at which income $\rho$ makes individual 2 indifferent between contributing and not contributing to public good $G$ (i.e., $g_{2}=0$ ). This transfer is called "an income-equalizing redistribution" from the rich to the poor, that is, $d \rho>0$. We denote individual 1's corresponding income level at this point by $\breve{\rho}$. At this point, we can further assume that while individual 1 still makes a positive contribution to public good $G$, individual 2 continues to contribute to only pubic good $H$ (which corresponds to the boundary point between Cases 2 and 7). ${ }^{9}$ From previous argument, the level of social welfare evaluated at this switching point is equal to that at the initial distribution of income $(\breve{\rho}, 1-\breve{\rho})$; that is,

$$
\begin{gathered}
W\left(u^{1}\left(\tilde{\rho}-g_{1}, g_{1}+g_{2}, h_{2}\right), u^{2}\left(1-\tilde{\rho}-h_{2}, g_{1}+g_{2}, h_{2}\right)\right) \\
\quad=W\left(u^{1}\left(\breve{\rho}-g_{1}, g_{1}, h_{2}\right), u^{2}\left(1-\breve{\rho}-h_{2}, g_{1}, h_{2}\right)\right) .
\end{gathered}
$$

Taking the right-hand side derivative of the right-hand side of $W$ in (11) with respect to $\rho$, evaluated at $\breve{\rho}$, we have

$$
\begin{gathered}
\left.\frac{d W}{d \rho}\right|_{\rho \rightarrow \breve{\rho}+}=W_{1} \frac{\partial u^{1}}{\partial x_{1}}\left[1-\frac{d g_{1}}{d \rho}+\frac{d g_{1}}{d(1-\rho)}\right]+W_{1} \frac{\partial u^{1}}{\partial G}\left[\frac{d g_{1}}{d \rho}-\frac{d g_{1}}{d(1-\rho)}\right] \\
+W_{1} \frac{\partial u^{1}}{\partial H}\left[\frac{d h_{2}}{d \rho}-\frac{d h_{2}}{d(1-\rho)}\right]+W_{2} \frac{\partial u^{2}}{\partial x_{2}}\left[-1-\frac{d h_{2}}{d \rho}+\frac{d h_{2}}{d(1-\rho)}\right] \\
+W_{2} \frac{\partial u^{2}}{\partial G}\left[\frac{d g_{1}}{d \rho}-\frac{d g_{1}}{d(1-\rho)}\right]+W_{2} \frac{\partial u^{2}}{\partial H}\left[\frac{d h_{2}}{d \rho}-\frac{d h_{2}}{d(1-\rho)}\right]
\end{gathered}
$$

where, we recall, $g_{1}=g_{1}(\rho, 1-\rho)$ and $h_{2}=h_{2}(1-\rho, \rho)$. Under non-identical preferences and from (9) and (10), we can rewrite (12) as follows:

$$
\begin{aligned}
\left.\frac{d W}{d \rho}\right|_{\rho \rightarrow \breve{\rho}+}= & W_{1} \frac{\partial u^{1}}{\partial x_{1}}\left[1+\frac{\partial u^{1} / \partial H}{\partial u^{1} / \partial x_{1}}\left(\frac{d h_{2}}{d \rho}-\frac{d h_{2}}{d(1-\rho)}\right)\right] \\
& +W_{2} \frac{\partial u^{2}}{\partial x_{2}}\left[-1+\frac{\partial u^{2} / \partial G}{\partial u^{2} / \partial x_{2}}\left(\frac{d g_{1}}{d \rho}-\frac{d g_{1}}{d(1-\rho)}\right)\right] .
\end{aligned}
$$

\footnotetext{
${ }^{9}$ Recall that individual 2 never stops contributing to public good $H$, because Assumption 1 does not allow for simultaneous zero supply of that public good. On the other hand, Case 8 (i.e., $g_{1}=0$ and $g_{2}>0$, $h_{1}>0$ and $h_{2}=0$ ) also never occurs because individual 1 should continue to contribute a positive amount of $g_{1}>0$, as his or her income is increasing. Note that Case 5 is possible, and we have already carried out this analysis.
} 
Although from Appendix A it follows that $d g_{1} / d \rho>0, d g_{1} / d(1-\rho)<0, d h_{2} / d \rho<0$, and $d h_{2} / d(1-\rho)>0\left(\right.$ we recall that $\left(\partial u^{1} / \partial H\right) /\left(\partial u^{1} / \partial x_{1}\right)<1$ for $h_{1}=0$ and $\left(\partial u^{2} / \partial G\right) /\left(\partial u^{2} / \partial x_{2}\right)<$ 1 for $\left.g_{2}=0\right)$, the signs of both the brackets on the right-hand side of (13) turn out to be ambiguous. ${ }^{10}$

Under identical preferences, in contrast, we can prove the following important lemma:

Lemma 2 Assuming that all individuals have identical preferences, neither Case 7 nor Case 8 holds.

Proof. First, we consider Case 7. Assume that the following inequalities hold:

$$
\begin{aligned}
& \frac{u_{G}^{1}\left(x_{1}, g_{1}, h_{2}\right)}{u_{x}^{1}\left(x_{1}, g_{1}, h_{2}\right)}=1>\frac{u_{G}^{2}\left(x_{2}, g_{1}, h_{2}\right)}{u_{x}^{2}\left(x_{2}, g_{1}, h_{2}\right)} \text { for } g_{1}>0 \text { and } g_{2}=0, \\
& \frac{u_{H}^{2}\left(x_{2}, g_{1}, h_{2}\right)}{u_{x}^{2}\left(x_{2}, g_{1}, h_{2}\right)}=1>\frac{u_{H}^{1}\left(x_{1}, g_{1}, h_{2}\right)}{u_{x}^{1}\left(x_{1}, g_{1}, h_{2}\right)} \text { for } h_{1}=0 \text { and } h_{2}>0 .
\end{aligned}
$$

(14) implies that $x_{2}>x_{1}$, while (15) implies that $x_{1}>x_{2}$ from the property of diminishing marginal rate of substitution between private consumption and the demand of the respective public goods (=strict quasi-concavity) in conjunction with identical individual preferences. This is clearly a contradiction. The proof for eliminating Case 8 follows in the same lines as with Case 7.

Lemma 2 implies that only Cases 1-6 can emerge under identical preferences so that the level of social welfare remains unchanged except when the distribution of income is extremely

${ }^{10}$ Alternatively, we can rewrite (13) as follows:

$$
\begin{aligned}
\left.\frac{d W}{d \rho}\right|_{\rho \rightarrow \hat{\rho}-}= & W_{1} \frac{\partial u^{1}}{\partial x_{1}}\left[1-\frac{\partial u^{1} / \partial G}{\partial u^{1} / \partial x_{1}} \frac{d g_{2}}{d(1-\rho)}-\right. \\
& \left.\frac{\partial u^{1} / \partial H}{\partial u^{1} / \partial x_{1}} \frac{d h_{2}}{d(1-\rho)}\right]-W_{2} \frac{\partial u^{2}}{\partial x_{2}}
\end{aligned}
$$

indicating that the income-equalizing redistribution policy (i.e., $d \rho>0$ ) is Pareto-improving if the following inequality holds:

$$
1-\frac{\partial u^{1} / \partial G}{\partial u^{1} / \partial x_{1}} \frac{d g_{2}}{d(1-\rho)}-\frac{\partial u^{1} / \partial H}{\partial u^{1} / \partial x_{1}} \frac{d h_{2}}{d(1-\rho)}>0 .
$$

Nevertheless, the sign of the above expression is still ambiguous, as stated in the text.

More specifically, we can show that under the Cobb-Douglas utility functions combined with some plausible parameter restrictions (e.g., Assumption 4), the level of social welfare unambiguously increases as a result of the income-equalizing redistribution policy; see Section 5 . 
unequal (i.e., Cases 5 and 6). Taken together, we have the first main result of this paper as follows:

Proposition 2 Assume that all individuals have identical and egoistic preferences in a twoindividual, two-public good economy, and the Nash equilibrium is stable. Now, we have the following:

(i) Starting from income distribution in which the rich contributes to all public goods while the poor does not contribute to any of them, the infinitesimal transfer of income from the poor to the rich (i.e., an inequality-raising redistribution) raises social welfare; and

(ii) any income redistribution policy does not affect social welfare unless the distribution of income is extremely unequal.

If we assume further that all individuals have non-identical and egoistic preferences, then we have as follows:

(iii) The inequality-raising redistribution described in statement (i) raises social welfare if the socially weighted marginal utility of the rich's private consumption plus the poor's socially weighted marginal utility of the two public goods are larger than that of the poor's private consumption; and, (iv) starting from the income distribution in which the rich contributes to all public goods while the poor contributes to only one public good, an infinitesimal transfer of income from the rich to the poor (i.e., an income-equalizing redistribution) may or may not raise social welfare.

Several remarks on Proposition 2 are in order here. First, we have generalized the result of Itaya et al. (1997) such that the income-inequality-raising redistribution policy raises social welfare in the presence of multiple voluntarily supplied public goods so long as the distribution of income is extremely unequal; the inequality of income should be praised in the economies with multiple voluntarily supplied goods as well. Note also that this seemingly paradoxical outcome remains valid irrespective of whether individual preferences are identical or non-identical. More precisely, it follows from statement $(i v)$ of Proposition 2 that under non-identical preferences, this conclusion holds true provided the gains in utility from the increased provision of both public goods as well as the increased private consumption of the rich, 
both of which are caused by income-inequality-raising redistribution, adequately compensate the loss due to reduced private consumption of the poor whereby social welfare is increased. Second, from statements $(i i)$ and $(i v)$ of Proposition 2, an income-equalizing redistribution policy raises the possibility that it may increase social welfare when individual preferences are non-identical and the distribution of income is not much unequal. In other words, under identical preferences, any redistribution policy is no longer effective, meaning that it does not affect social welfare at all. These conflicting results arise because under identical preferences, all individuals take the same pattern of contribution to public goods so that the case where each individual contributes to a different public good, such as in Cases 7 and 8, never arises, inevitably leading to the neutrality property. Thus, social welfare ends up being invariant with respect to any change in distribution of income. Third, the main point emerging from this discussion is that whether a redistribution policy is effective or not crucially hinges on the individual preferences being identical or non-identical. More specifically, when the distribution of income is not much unequal, whether social welfare remains constant or is potentially increasing with respect to the income-equalizing redistribution policy hinges on the individual preferences being identical or non-identical. Fourth, we have proved only the local properties with regard to the effect on social welfare in the neighborhood of the switching point where individuals change their patterns of contributions, because we focus on an infinitesimal redistribution policy of income. To analyze the "global" effect on social welfare, we need to examine numerical examples by employing specific utility functions such as the Cobb-Douglas preferences, which will be discussed in the following sections.

\section{Caring Preferences}

Assuming caring preferences, each individual has the following utility function:

$$
U^{i}\left(x_{i}, G, H\right) \equiv u^{i}\left(x_{i}, G, H\right)+\alpha_{i} u^{j}\left(x_{j}, G, H\right), i, j=1,2 ; i \neq j,
$$

where $\alpha_{i}$ represents the weight attached to the other individual with $\alpha_{i}<1$ for $i=1,2$. We refer to (16) as a social utility function of individual $i$, in that one has altruism toward 
another individual's utility. Note as well that the non-negative altruism parameters $\alpha_{i}$ should be less than 1, implying that individuals care for others, but not as much as they do care for themselves.

The additional Kuhn-Tucker first-order conditions for individual $i^{\prime} \mathrm{s}(i=1,2)$ optimal income transfers are given by

$$
\begin{gathered}
-u_{x}^{i}\left(m_{i}-g_{i}-h_{i}-t_{i}+t_{j}, G, H\right)+\alpha_{i} u_{x}^{j}\left(m_{j}-g_{j}-h_{j}-t_{j}+t_{i}, G, H\right) \leq 0 \\
\text { for } t_{i} \geq 0, i=1,2 ; i \neq j
\end{gathered}
$$

where $t_{i}$ and $t_{j}$ represent the transfers from individual $i$ to $j$ and from $j$ to $i$ respectively.

In Cases 1-4, the neutrality theorem remains valid independent of whether the value of $t_{i}$ is positive or zero. Thus, in these cases, social welfare remains invariant with respect to the distribution of income as before. We next focus on Cases 5-8, where the neutrality theorem ceases to be valid in the previous model without altruistic preferences.

Before doing this, we first prove the following lemma:

Lemma 3 Both individuals never make a positive transfer to each other simultaneously.

Proof. Suppose first that the contrary holds in Case 7; that is, $t_{1}>0$ and $t_{2}>0$, implying that

$$
\begin{aligned}
& -u_{x}^{1}\left(m_{1}-g_{1}-t_{1}+t_{2}, g_{1}, h_{2}\right)+\alpha_{1} u_{x}^{2}\left(m_{2}-h_{2}-t_{2}+t_{1}, g_{1}, h_{2}\right)=0 \\
& -u_{x}^{2}\left(m_{2}-h_{2}-t_{2}+t_{1}, g_{1}, h_{2}\right)+\alpha_{2} u_{x}^{1}\left(m_{1}-g_{1}-t_{1}+t_{2}, g_{1}, h_{2}\right)=0 .
\end{aligned}
$$

By substituting the second expression into the first expression, we obtain

$$
u_{x}^{1}(.)=\alpha_{1} u_{x}^{2}(.)=\alpha_{1} \alpha_{2} u_{x}^{1}(,)
$$

However, the above equalities boil down to $1=\alpha_{1} \alpha_{2}$, which apparently contradicts the assumption that $\alpha_{1} \alpha_{2}<1$. Similarly, we can demonstrate that Lemma 3 holds for Cases 5,6 , and 8 as well. 
Thus, we can rule out the case where $t_{1}>0$ and $t_{2}>0$; consequently, there remain only three cases, where (i) $t_{1}>0$ and $t_{2}=0$, (ii) $t_{1}=0$ and $t_{2}>0$, and (iii) $t_{1}=0$ and $t_{2}=0$. Since the last case, (iii), is equivalent to the case of egoistic preferences, which we already investigated in the previous section, we will focus on the following eight possibilities:

(A) $t_{1}>0$ and $t_{2}=0$ in Cases $5-8$, as listed in subsection 2.1,

(B) $t_{1}=0$ and $t_{2}>0$ in Cases $5-8$, as listed in subsection 2.1.

Cornes et al. (2012) further extend the concept of linked individuals defined by Definition 2 to the individuals connected with one another through positive voluntary income transfers and/or through positive contributions to the same public good, and further demonstrate that the redistribution of income does not affect real allocations among non-contributors to public goods if voluntary income transfers occur between them; thus, the neutrality theorem continues to hold so that the level of social welfare remains constant in terms of distribution of income. Formally, they have given a slightly generalized definition for "linked individuals" compared to Definition 2:

Definition 3 Individuals $i$ and $i^{\prime}$ are linked at an equilibrium if at least one of the following conditions holds: (i) positive transfers exist between $i$ and $i^{\prime}$; (ii) they contribute to the same public good.

Under this definition, Cornes et al. (2012) demonstrate the following lemma:

Lemma 4 (Corollary 1 of Cornes et al., 2012) An income redistribution restricted to a set of linked individuals and that maintains the links between them has no effect on the original equilibrium allocation. Moreover, if every individual is linked through at least one link supported by positive voluntary income transfers to other individuals or positive private contributions to the public good, neutrality holds.

In light of Lemma 4 , if individuals $h$ and $h+k$ are linked, so too are any two individuals belonging to the same chain, who are also neutral with a redistribution of income due to the presence of one-way income transfers (i.e., either $t_{1}=0$ and $t_{2}>0$ or $t_{1}>0$ and $t_{2}=0$ ). In 
order to apply Lemma 4, we need to also identify whether or not voluntary income transfers occur between individuals in Cases 5-8 and to which public good each individual contributes. We have the following lemma:

Lemma 5 When individuals 1 and 2 have altruistic preferences toward each other, we have the following results:

(i) In Case 5, individual 2 makes positive voluntary income transfers to 1, whereas individual 1 does not;

(ii) In Case 6, individual 1 makes positive voluntary income transfers to 2, whereas 2 does not; and

(iii) In Cases 7 and 8, neither of the individuals makes any voluntary income transfers to each other.

Proof. The proofs are given in Appendix B.

In Case 5, where only individual 2 makes positive contributions to both public goods, individual 2 could be viewed as being richer than 1, and so it would be natural to expect voluntary income transfers from individual 2 to 1 . The opposite holds true in Case 6 for the same reasoning.

On the other hand, although one may expect the neutrality theorem in Cases 7 and 8 to be valid owing to positive voluntary income transfers, this may not hold true; indeed, the redistribution policy does affect social welfare. This is because when the income levels of individuals are not as much different as in Cases 7 and 8, the marginal utility from transferring income to others is relatively weak compared to Cases 5 and 6 so that an individual benefits more from contributing to his or her preferred public goods rather than making voluntary income transfers to others, which ceases the voluntary income transfers to other individuals.

To sum up, we have the second main result as follows:

Proposition 3 The results in statements (i)-(iv) of Proposition 2 continue to hold in the presence of altruistic preferences unless the income level of a non-contributor is sufficiently 
low. If it is sufficiently low, the redistribution of income between those individuals does not affect social welfare.

\section{Example 1: Identical Cobb-Douglas Preferences}

So far, we considered the local properties of the effects of income inequality on social welfare, in that the welfare analysis is limited to the neighborhood of the switching point of the distribution of income at which the profiles of individual contributions are changed. In this section, we examine a case where each individual maximizes the following identical CobbDouglas utility function:

$$
\begin{array}{ll} 
& U^{i}\left(x_{i}, G, H\right) \equiv \ln x_{i}+\beta_{1} \ln G+\beta_{2} \ln H+\alpha\left[\ln x_{j}+\beta_{1} \ln G+\beta_{2} \ln H\right], \\
\text { s. t. } & x_{i}+g_{i}+h_{i}+t_{i}=m_{i}+t_{j}, \quad g_{i} \geq 0, h_{i} \geq 0 \text { and } t_{i}, t_{j} \geq 0, i, j=1,2 ; i \neq j,
\end{array}
$$

where $G \equiv g_{1}+g_{2}, H \equiv h_{1}+h_{2}, m_{1} \equiv \rho$, and $m_{2} \equiv 1-\rho$ (i.e., the income levels of both individuals are different).

This specification allows us not only to further sharpen our general results obtained in the previous sections, but also to draw a diagram, in particular, as to the shape of the social welfare function over the whole range of income distribution rather than a local behavior of the social welfare function. Note that no corner solution for private consumption arises (i.e., $\left.x_{i}=\rho-g_{i}-h_{i}>0\right)$ from the features of the Cobb-Douglas utility function.

For analytical simplicity, we consider the utilitarian (symmetric) social welfare function, such as $W=U^{1}+U^{2}$, which reflects the case where two individuals are equally important and nothing is modified so long as the social welfare function is symmetric. To facilitate the analysis, we further make the following harmless assumption:

Assumption 3 The utility functions $U^{i}\left(x_{i}, G, H\right)$ of $i=1,2$ in (17) satisfy $(i) 0<\beta_{2}<\beta_{1}<$ 1 and $($ ii $) 0<\alpha<1$.

Assumption 3 (i) implies that both individuals prefer the public good $G$ to $H$. From solving 
this problem, we have the following first-order conditions:

$$
\begin{aligned}
\frac{x_{i} \beta_{1}}{G}(1+\alpha) & \leq 1 \quad \text { with equality if } g_{i}>0, i=1,2, \\
\frac{x_{i} \beta_{2}}{H}(1+\alpha) & \leq 1 \quad \text { with equality if } h_{i}>0, i=1,2, \\
\frac{x_{1}}{x_{2}} \alpha & \leq 1 \quad \text { with equality if } t_{1}>0, \\
\frac{x_{2}}{x_{1}} \alpha & \leq 1 \quad \text { with equality if } t_{2}>0 .
\end{aligned}
$$

\subsection{Egoistic Preferences}

We first consider the case where each individual does not have altruism toward the other; that is, $\alpha=0$ for both individuals in (17). Since this implies that each individual does not make transfers to the other, we have $t_{i}=0, i=1,2$ in (18). Elementary manipulations of (19) (setting $\alpha=0$ and thus dropping the third and fourth equations in (19)) reveal that, depending on the distribution of income, the Nash equilibrium may fall into any of the three regimes based on the pattern of equilibrium allocation as illustrated in Figure 1. Table 1 summarizes the regimes:

\begin{tabular}{|c|c|c|c|c|c|}
\hline Regime & Income distribution & $x_{1}$ & $x_{2}$ & $G$ & $H$ \\
\hline I & $0 \leqq \rho \leqq \frac{1}{1+X}$ & $\rho$ & $\frac{1-\rho}{X}$ & $\frac{\beta_{1}(1-\rho)}{X}$ & $\frac{\beta_{2}(1-\rho)}{X}$ \\
\hline II & $\frac{1}{1+X} \leqq \rho \leqq \frac{X}{1+X}$ & $\frac{1}{1+X}$ & $\frac{1}{1+X}$ & $\frac{\beta_{1}}{1+X}$ & $\frac{\beta_{2}}{1+X}$ \\
\hline III & $\frac{X}{1+X} \leqq \rho \leqq 1$ & $\frac{\rho}{X}$ & $1-\rho$ & $\frac{\beta_{1} \rho}{X}$ & $\frac{\beta_{2} \rho}{X}$ \\
\hline
\end{tabular}

Table 1 . The profile of equilibrium allocation where $X \equiv 1+\beta_{1}+\beta_{2}$

Furthermore, Figure 2 shows how the level of social welfare varies according to the distribution of income. The intuitive exposition is as follows. In Regime $I$ of Table 1, where individual 2 has most (or all) of the income, the levels of both public goods provision correspond to his most preferred one, because he is the sole contributor to both public goods; hence, the resulting outcome is Pareto efficient. As increases in the income share of individual 1 lead her to spend more on private consumption good, it leads individual 2 to spend less on the two 
public goods and on private consumption. Accordingly, social welfare initially rises, because the marginal utility of individual 1's private consumption is very high when her income level is extremely low. As the marginal utility of her private consumption falls with her income share, social welfare starts falling after the point at which the marginal utilities of private consumption and the two public goods are equal.

When the income of individual 1 is increased to the level of Regime $I I$, she starts contributing to both public goods in Regime $I I$. Thus, the aggregate quantities of the two public goods do not depend on the distribution of income, and thus social welfare remains constant in Regime $I I$ because the neutrality theorem holds (recall Proposition 1). As stated in the standard literature, however, those aggregate quantities are inefficiently low due to the secondbest property of the Nash equilibrium. Note not only that under the identical Cobb-Douglas preferences, Cases 7 and 8 vanish (recall Lemma 2), but also that individual contributions are indeterminate in Regime $I I$, even though the equilibrium levels of $G$ and $H$ are uniquely determined, which is consistent with Proposition 2 of Cornes and Itaya (2000) (see also footnote $3)$.

As we continue to transfer income from individual 2 to individual 1 , the income level of individual 2 falls to the point at which he stops contributing to both public goods, and then we move to Regime $I I I$. Regime $I I I$ is analogous to Regime $I$, with individuals 1 and 2 exchanged. ${ }^{11}$

\subsection{Caring Preferences}

Finally, we examine the case of altruistic preferences where each individual has altruism toward the other and is willingly to make transfers to the other. That is, $U^{1} \equiv u^{1}\left(x_{1}, G, H\right)+$ $\alpha u^{2}\left(x_{2}, G, H\right), U^{2} \equiv u^{2}\left(x_{2}, G, H\right)+\alpha u^{1}\left(x_{1}, G, H\right)$. Elementary manipulations reveal that, depending on the distribution of income, the Nash equilibrium falls into any one of five regimes

\footnotetext{
${ }^{11}$ Note also that the higher the preference parameter (i.e., $\beta_{1}$ or $\beta_{2}$ ) toward either public good, the wider is the range of Regime $I I$ where individuals 1 and 2 contribute to both public goods.
} 


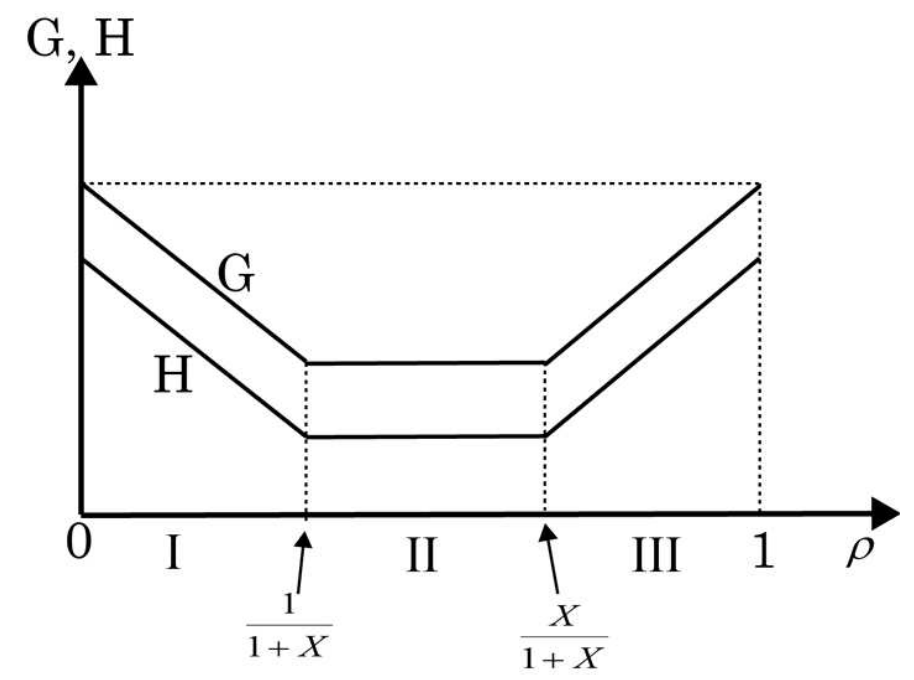

Figure 1: Aggregate quantities of the two public goods under egoistic and identical CobbDouglas preferences

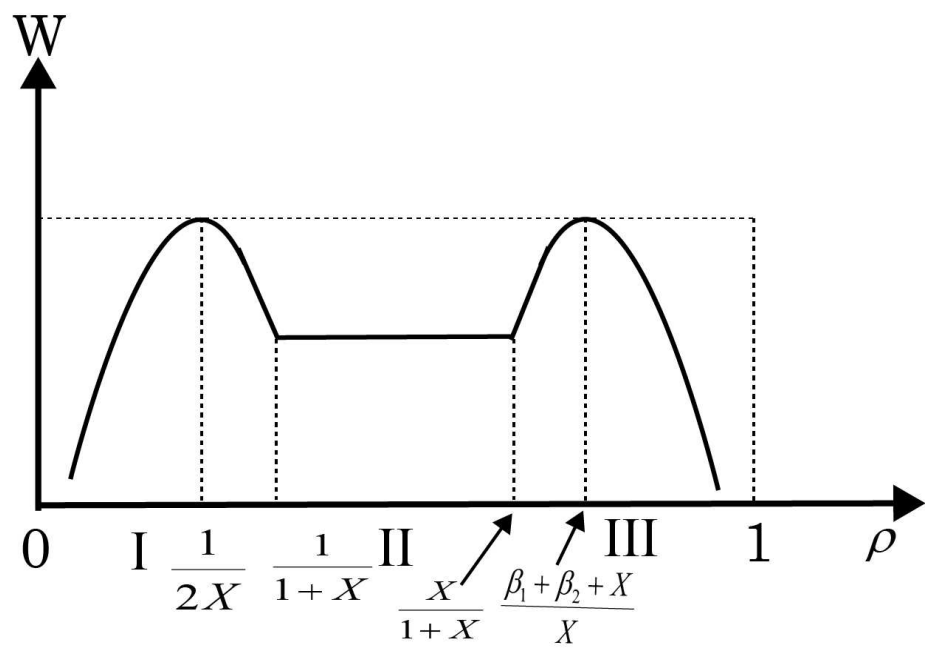

Figure 2: Social Welfare under egoistic and indentical Cobb-Douglas preferences 
based on the pattern of real allocations. Table 2 summarizes the regimes:

\begin{tabular}{|c|c|c|c|c|c|}
\hline Regime & Income distribution & $x_{1}$ & $x_{2}$ & $G$ & $H$ \\
\hline I & $0 \leqq \rho \leqq \frac{\alpha}{(1+\alpha) X}$ & $\frac{\alpha}{(1+\alpha) X}$ & $\frac{1}{(1+\alpha) X}$ & $\frac{\beta_{1}}{X}$ & $\frac{\beta_{2}}{X}$ \\
\hline II & $\frac{\alpha}{(1+\alpha) X} \leqq \rho \leqq \rho_{I I}$ & $\rho$ & $\frac{1-\rho}{\alpha\left(\beta_{1}+\beta_{2}\right)+X}$ & $\frac{\beta_{1}(1+\alpha)(1-\rho)}{\alpha\left(\beta_{1}+\beta_{2}\right)+X}$ & $\frac{\beta_{2}(1+\alpha)(1-\rho)}{\alpha\left(\beta_{1}+\beta_{2}\right)+X}$ \\
\hline III & $\rho_{I I}<\rho \leqq \rho_{I I I}$ & $\frac{1}{1+\alpha\left(\beta_{1}+\beta_{2}\right)+X}$ & $\frac{1}{1+\alpha\left(\beta_{1}+\beta_{2}\right)+X}$ & $\frac{\beta_{1}(1+\alpha)}{1+\alpha\left(\beta_{1}+\beta_{2}\right)+X}$ & $\frac{\beta_{2}(1+\alpha)}{1+\alpha\left(\beta_{1}+\beta_{2}\right)+X}$ \\
\hline IV & $\rho_{I I I} \leqq \rho \leqq \frac{\alpha\left(\beta_{1}+\beta_{2}\right)+X}{(1+\alpha) X}$ & $\frac{\rho}{\alpha\left(\beta_{1}+\beta_{2}\right)+X}$ & $1-\rho$ & $\frac{\beta_{1}(1+\alpha) \rho}{\alpha\left(\beta_{1}+\beta_{2}\right)+X}$ & $\frac{\beta_{2}(1+\alpha) \rho}{\alpha\left(\beta_{1}+\beta_{2}\right)+X}$ \\
\hline V & $\frac{\alpha\left(\beta_{1}+\beta_{2}\right)+X}{(1+\alpha) X} \leqq \rho \leqq 1$ & $\frac{1}{(1+\alpha) X}$ & $\frac{\alpha}{(1+\alpha) X}$ & $\frac{\beta_{1}}{X}$ & $\frac{\beta_{2}}{X}$ \\
\hline
\end{tabular}

Table 2. The profile of equilibrium allocation

$$
\text { where } \rho_{I I} \equiv \frac{1}{1+\alpha\left(\beta_{1}+\beta_{2}\right)+X} \text { and } \rho_{I I I} \equiv \frac{\alpha\left(\beta_{1}+\beta_{2}\right)+X}{1+\alpha\left(\beta_{1}+\beta_{2}\right)+X} \text {. }
$$

One notable feature of Table 2 is that in Regimes $I, I I I$, and $V$, the aggregate quantities of the two public goods as well as the private consumption of both individuals do not depend on the values of $\rho$; consequently, the neutrality theorem holds and thus social welfare remains constant. Figure 3 illustrates the behavior of equilibrium provisions of the two public goods as the income distribution varies.

Figure 4 illustrates the shape of the social welfare function. We make several remarks. First, if one individual has most (or all) of the income, then she or he will make a transfer to the poorer as well as be the sole contributor to both public goods. This feature adds flat segments at the extremes of Figures 1 and 2, as shown in Figures 3 and 4 (which are denoted by Regimes $I$ and $V$ ), respectively. This is because, for example, if individual 2 has the most income (i.e., $\rho<\alpha /(1+\alpha) X)$, then 2's altruistic motivation will be operating and thus 2 is willing to make voluntary transfers to 1 , and, at the same time, is the sole contributor to both public goods, and vice versa if individual 1 has most income (i.e., $\left.\left[\alpha\left(\beta_{1}+\beta_{2}\right)+X\right] /(1+\alpha) X<\rho\right)$. From Lemma 4, in either case, voluntary income transfers will generate the neutrality property in Regimes $I$ and $V$, so that social welfare remains constant. Moreover, the outcomes in Regimes $I$ and $V$ (in addition to Regimes $I I$ and $I V$ ) are Pareto-efficient, because individual 2 in Regimes $I$ and $I I$ ( 1 in Regimes $I V$ and $V$ ) is an effective dictator, so that any feasible 


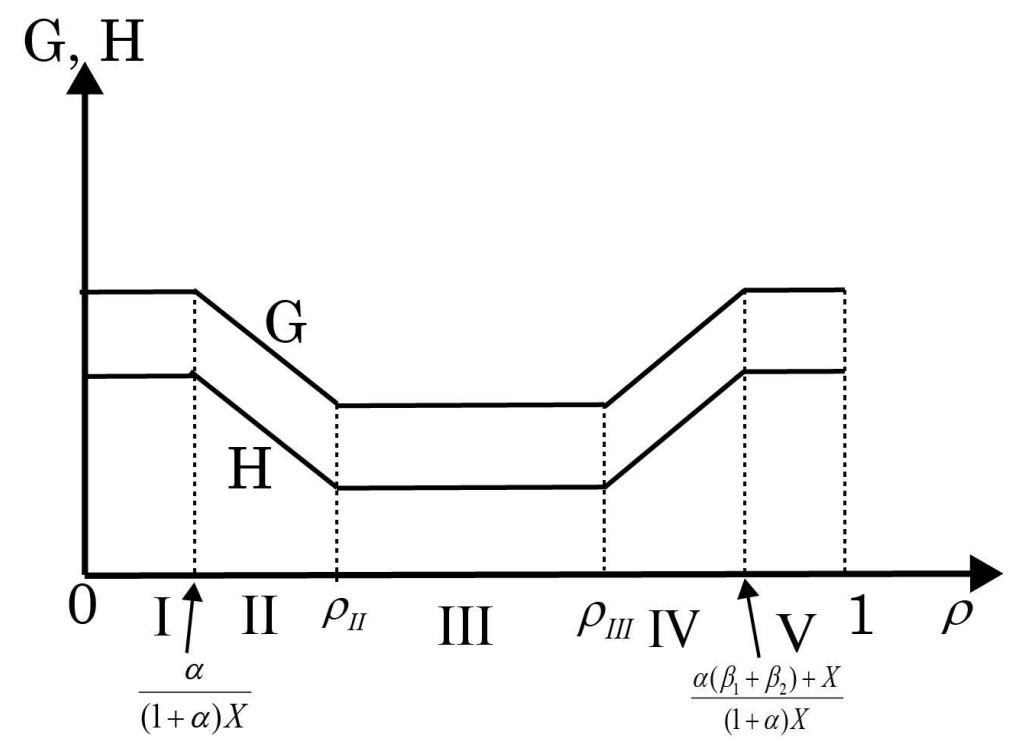

Figure 3: Aggregate quantities of the two public goods under altruistic and identical CobbDouglass preferences

allocation will make 2 (1) worse off. ${ }^{12}$

\section{Example 2: Non-identical Cobb-Douglas Preferences}

In this section, we consider the case where each individual solves the following problem under non-identical Cobb-Douglas preferences:

$$
\begin{array}{r}
\max _{\left\{x_{1}, g_{1}, h_{1}, t_{1}\right\}} U^{1}=\log x_{1}+\beta_{1} \log G+\beta_{2} \log H+\alpha_{1}\left[\log x_{2}+\delta_{1} \log G+\delta_{2} \log H\right], \\
\text { s.t. } x_{1}+g_{1}+h_{1}+t_{1}=\rho+t_{2}, g_{1} \geq 0, h_{1} \geq 0 \text { and } t_{1} \geq 0, \\
\max _{\left\{x_{2}, g_{2}, h_{2}, t_{1}\right\}} U^{2}=\log x_{2}+\delta_{1} \log G+\delta_{2} \log H+\alpha_{2}\left[\log x_{1}+\beta_{1} \log G+\beta_{2} \log H\right], \\
\text { s.t. } \quad x_{2}+g_{2}+h_{2}+t_{2}=1-\rho+t_{1}, g_{2} \geq 0, h_{2} \geq 0 \text { and } t_{2} \geq 0 .
\end{array}
$$

\footnotetext{
${ }^{12}$ We may also note that the size of these ranges depends on the degree of altruism; more precisely, if each individual cares more about the other individual (i.e., the common altruistic parameter $\alpha$ becomes larger), Regimes $I$ and $V$ both become wider.
} 


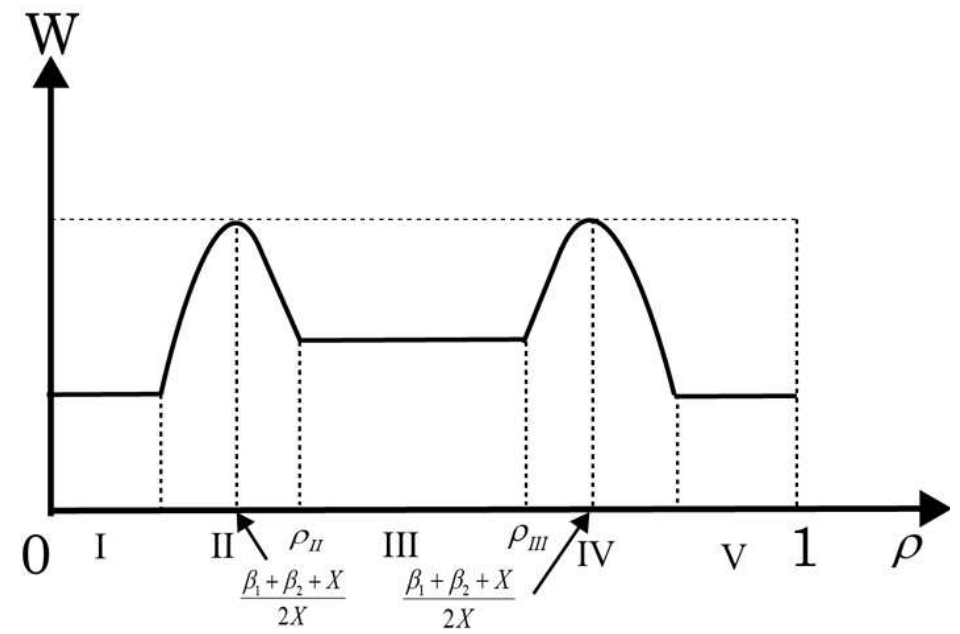

Figure 4: Social welfare under altruistic and identical Cobb-Douglass preferences, where $\rho_{I I} \equiv$ $\frac{1}{1+\alpha\left(\beta_{1}+\beta_{2}\right)+X}$ and $\rho_{I I I} \equiv \frac{\alpha\left(\beta_{1}+\beta_{2}\right)+X}{1+\alpha\left(\beta_{1}+\beta_{2}\right)+X}$.

Solving this problem, we have the following first-order conditions:

$$
\begin{gathered}
\frac{x_{1}}{G}\left(\beta_{1}+\alpha_{1} \delta_{1}\right) \leq 1 \quad \text { with equality if } g_{1} \geq 0, \\
\frac{x_{1}}{H}\left(\beta_{2}+\alpha_{1} \delta_{2}\right) \leq 1 \quad \text { with equality if } h_{1} \geq 0, \\
\quad \frac{x_{2}}{x_{1}} \alpha_{1} \leq 1 \quad \text { with equality if } t_{1} \geq 0, \\
\frac{x_{2}}{G}\left(\delta_{1}+\alpha_{2} \beta_{1}\right) \leq 1 \quad \text { with equality if } g_{2} \geq 0, \\
\frac{x_{2}}{H}\left(\delta_{2}+\alpha_{2} \beta_{2}\right) \leq 1 \quad \text { with equality if } h_{2} \geq 0, \\
\frac{x_{2}}{x_{1}} \alpha_{2} \leq 1 \text { with equality if } t_{2} \geq 0 .
\end{gathered}
$$

We make the following assumption in order to avoid unnecessary complications:

Assumption 4 The Cobb-Douglas utility functions $u^{i}\left(x_{i}, G, H\right)$ for $i=1,2$ satisfy (i) $\delta_{1}<$ $\beta_{2}<\delta_{2}<\beta_{1}<1$ and (ii) $\beta_{1}<2 \delta_{1}$.

Assumption 4 (i) implies that individual 1 prefers public good $G$ to $H$, while individual 2 prefers public good $H$ to $G$. Assumption 4 (ii) gives an upper bound on the strength of the preferences of individual 1 toward public good $G$. 


\subsection{Egoistic Preferences}

We first examine the case where each individual does not have altruistic preferences toward the other (i.e., setting $\alpha_{1}=\alpha_{2}=0$ in (20) and (22), and thus $t_{1}=t_{2}=0$ in (21) and (23)). Elementary manipulations of (24) reveal that, depending on the distribution of income, the Nash equilibrium may fall into any one of five regimes listed in Table 3 as to the pattern of equilibrium allocations:

\begin{tabular}{|c|c|c|c|c|c|}
\hline Regime & Income distribution & $x_{1}$ & $x_{2}$ & $G$ & $H$ \\
\hline I & $0 \leqq \rho \leqq \frac{\delta_{1}}{\delta_{1}+\beta_{1} D}$ & $\rho$ & $\frac{1-\rho}{D}$ & $\frac{\delta_{1}(1-\rho)}{D}$ & $\frac{\delta_{2}(1-\rho)}{D}$ \\
\hline II & $\frac{\delta_{1}}{\delta_{1}+\beta_{1} D} \leqq \rho \leqq \frac{\delta_{1}\left(1+\beta_{1}\right)}{\delta_{1}+\beta_{1} D}$ & $\frac{\delta_{1}}{\delta_{1}+\beta_{1} D}$ & $\frac{\beta_{1}}{\delta_{1}+\beta_{1} D}$ & $\frac{\beta_{1} \delta_{1}}{\delta_{1}+\beta_{1} D}$ & $\frac{\beta_{1} \delta_{2}}{\delta_{1}+\beta_{1} D}$ \\
\hline III & $\frac{\delta_{1}\left(1+\beta_{1}\right)}{\delta_{1}+\beta_{1} D} \leqq \rho \leqq \frac{\delta_{2}\left(1+\beta_{1}\right)}{\beta_{2}+\delta_{2} X}$ & $\frac{\rho}{1+\beta_{1}}$ & $\frac{1-\rho}{1+\delta_{2}}$ & $\frac{\beta_{1} \rho}{1+\beta_{1}}$ & $\frac{\delta_{2}(1-\rho)}{1+\delta_{2}}$ \\
\hline IV & $\frac{\delta_{2}\left(1+\beta_{1}\right)}{\beta_{2}+\delta_{2} X} \leqq \rho \leqq \frac{\delta_{2} X}{\beta_{2}+\delta_{2} X}$ & $\frac{\delta_{2}}{\beta_{2}+\delta_{2} X}$ & $\frac{\beta_{2}}{\beta_{2}+\delta_{2} X}$ & $\frac{\beta_{1} \delta_{2}}{\beta_{2}+\delta_{2} X}$ & $\frac{\beta_{2} \delta_{2}}{\beta_{2}+\delta_{2} X}$ \\
\hline $\mathrm{V}$ & $\frac{\delta_{2} X}{\beta_{2}+\delta_{2} X} \leqq \rho \leqq 1$ & $\frac{\rho}{X}$ & $1-\rho$ & $\frac{\beta_{1} \rho}{X}$ & $\frac{\beta_{2} \rho}{X}$ \\
\hline
\end{tabular}

Table 3. The profile of equilibrium allocation where $D \equiv 1+\delta_{1}+\delta_{2}$

An inspection of Table 3 reveals that in Regimes $I I$ and $I V$, the aggregate quantities of the two public goods do not depend on the distribution of income because of the neutral property, and so the level of social welfare remains invariant. Figure 5 illustrates the total provision of the two public goods in terms of distribution of income. One notable feature of Table 3 and Figure 5 is that the points at which flat segments begin are the same for the two goods despite heterogeneous preferences among individuals.

We are now ready to analyze how the inequality of income affects the level of social welfare. Taken together, under Assumption 4, we can illustrate the shape of the social welfare function following Regime III of Figure 6. Formally, it follows from (13) that

$$
\begin{aligned}
\left.\frac{d W}{d \rho}\right|_{\rho \rightarrow \breve{\rho}+} & =\frac{1}{x_{1}}\left[1+\beta_{2} \frac{x_{1}}{H}\left(-\frac{d h_{2}}{d(1-\rho)}\right)\right]+\frac{1}{x_{2}}\left[-1+\delta_{1} \frac{x_{2}}{G} \frac{d g_{1}}{d \rho}\right] \\
& =\frac{1+\beta_{1}}{\rho}-\frac{\beta_{2}}{1-\rho}-\frac{1+\delta_{2}}{1-\rho}+\frac{\delta_{1}}{\rho}
\end{aligned}
$$

where $d g_{1} / d \rho=\beta_{1} /\left(1+\beta_{1}\right)>0, d h_{2} / d(1-\rho)=\delta_{2} /\left(1+\delta_{2}\right)>0, x_{1}=\rho /\left(1+\beta_{1}\right)$, and 


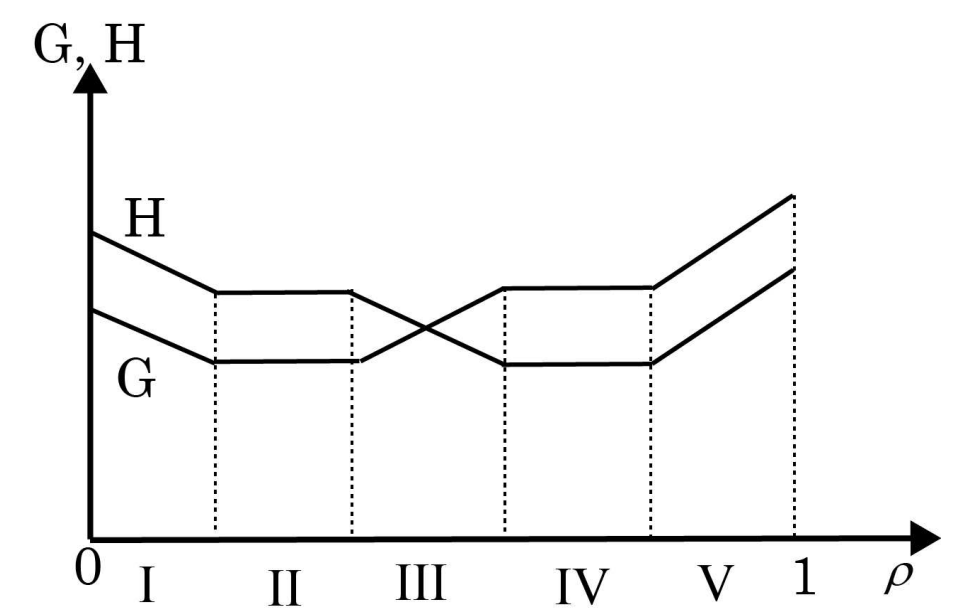

Figure 5: The aggregate quantities of the two public goods under altruistic and non-identical preferences

$x_{2}=(1-\rho) /\left(1+\delta_{2}\right)$. Substituting $\breve{\rho}=\delta_{1}\left(1+\beta_{1}\right) /\left(\delta_{1}+\beta_{1} D\right)$, which corresponds to the left-hand side boundary point of Regime III, for $\rho$ in (25) yields

$$
d W /\left.d \rho\right|_{\rho \rightarrow \breve{\rho}+}=\frac{\left(\delta_{1}+\beta_{1} D\right)}{\delta_{1}\left(1+\beta_{1}\right)\left(\beta_{1} D-\beta_{1} \delta_{1}\right)}\left[\left(1+\beta_{1}\right)\left(\beta_{1}+\beta_{1} \delta_{2}-\delta_{1} \beta_{2}\right)-\delta_{1}\left(1+\delta_{2}\right)\right]>0
$$

while substituting $\breve{\rho}=\delta_{2}\left(1+\beta_{1}\right) /\left(\beta_{2}+\delta_{2} X\right)$, which corresponds to the right-hand side boundary point of Regime III, for $\rho$ in (25) yields

$$
d W /\left.d \rho\right|_{\rho \rightarrow \breve{\rho}-}=\frac{\left(\beta_{2}+\delta_{2} X\right)}{\delta_{2}\left(1+\beta_{1}\right) \beta_{2}\left(1+\delta_{2}\right)}\left[-\left(1+\delta_{2}\right)\left[\beta_{1} \delta_{2}-\delta_{1} \beta_{2}+\delta_{2}\right]+\beta_{2}\left(1+\beta_{1}\right)\right]<0
$$

We can easily see that the signs of (26) and (27) follow from Assumption 4. In other words, Assumption 4 is a sufficient condition to derive an inverse $U$-shaped graph of the social welfare function over the middle range of income distribution (i.e., Regime $I I I$ ). We draw the shape of the social welfare function in Figure 6 when $\beta_{1}=0.8, \beta_{2}=0.6, \delta_{1}=0.6$, and $\delta_{2}=0.7$. 


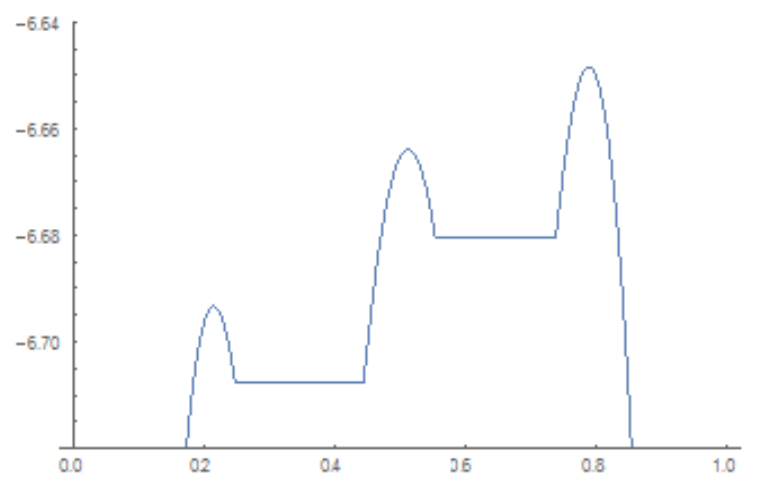

Figure 6: Social welfare under altruistic and non-identical Cobb-Douglass preferences

\subsection{Caring Preferences}

Finally, we examine the case of altruistic preferences characterized by (20) and (22). Table 5 summarizes the equilibrium profiles of allocations:

\begin{tabular}{|c|c|c|c|c|c|}
\hline Regime & Income distribution & $x_{1}$ & $x_{2}$ & $G$ & $H$ \\
\hline I & $0 \leqq \rho \leqq \frac{\alpha_{2}}{D+\alpha_{2} X}$ & $\frac{\alpha_{2}}{D+\alpha_{2} X}$ & $\frac{1}{D+\alpha_{2} X}$ & $\frac{\delta_{1}+\alpha_{2} \beta_{1}}{D+\alpha_{2} X}$ & $\frac{\delta_{2}+\alpha_{2} \beta_{2}}{D+\alpha_{2} X}$ \\
\hline II & $\frac{\alpha_{2}}{D+\alpha_{2} X} \leqq \rho \leqq \frac{\delta_{1}+\alpha_{2} \beta_{1}}{K}$ & $\rho$ & $\frac{1-\rho}{\alpha_{2}\left(\beta_{1}+\beta_{2}\right)+D}$ & $\frac{\left(\delta_{1}+\alpha_{2} \beta_{1}\right)(1-\rho)}{\alpha_{2}\left(\beta_{1}+\beta_{2}\right)+D}$ & $\frac{\left(\delta_{2}+\alpha_{2} \beta_{2}\right)(1-\rho)}{\alpha_{2}\left(\beta_{1}+\beta_{2}\right)+D}$ \\
\hline III & $\frac{\delta_{1}+\alpha_{2} \beta_{1}}{K}<\rho \leqq \rho_{I I I}$ & $\frac{\delta_{1}+\alpha_{2} \beta_{1}}{K}$ & $\frac{\beta_{1}+\alpha_{1} \delta_{1}}{K}$ & $\frac{\left(\beta_{1}+\alpha_{1} \delta_{1}\right)\left(\delta_{1}+\alpha_{2} \beta_{1}\right)}{K}$ & $\frac{\left(\beta_{1}+\alpha_{1} \delta_{1}\right)\left(\delta_{2}+\alpha_{2} \beta_{2}\right)}{K}$ \\
\hline IV & $\rho_{I I I} \leqq \rho \leqq \rho_{I V}$ & $\frac{\rho}{1+\beta_{1}+\alpha_{1} \delta_{1}}$ & $\frac{1-\rho}{1+\delta_{2}+\alpha_{2} \beta_{2}}$ & $\frac{\left(\beta_{1}+\alpha_{1} \delta_{1}\right) \rho}{1+\beta_{1}+\alpha_{1} \delta_{1}}$ & $\frac{\left(\delta_{2}+\alpha_{2} \beta_{2}\right)(1-\rho)}{1+\delta_{2}+\alpha_{2} \beta_{2}}$ \\
\hline V & $\rho_{I V} \leqq \rho \leqq \rho_{V}$ & $\frac{\delta_{2}+\alpha_{2} \beta_{2}}{L}$ & $\frac{\beta_{2}+\alpha_{1} \delta_{2}}{L}$ & $\frac{\left(\beta_{1}+\alpha_{1} \delta_{1}\right)\left(\delta_{2}+\alpha_{2} \beta_{2}\right)}{L}$ & $\frac{\left(\beta_{2}+\alpha_{1} \delta_{2}\right)\left(\delta_{2}+\alpha_{2} \beta_{2}\right)}{L}$ \\
\hline VI & $\rho_{V} \leqq \rho \leqq X+\alpha_{1}\left(\delta_{1}+\delta_{2}\right)$ \\
$X+\alpha_{1} D$ & $\frac{\rho}{X+\alpha_{1}\left(\delta_{1}+\delta_{2}\right)}$ & $1-\rho$ & $\frac{\left(\beta_{1}+\alpha_{1} \delta_{1}\right) \rho}{X+\alpha_{1}\left(\delta_{1}+\delta_{2}\right)}$ & $\frac{\left(\beta_{2}+\alpha_{1} \delta_{2}\right) \rho}{X+\alpha_{1}\left(\delta_{1}+\delta_{2}\right)}$ \\
\hline VII & $\frac{X+\alpha_{1}\left(\delta_{1}+\delta_{2}\right)}{X+\alpha_{1} D} \leqq \rho \leqq 1$ & $\frac{1}{X+\alpha_{1} D}$ & $\frac{\alpha_{1}}{X+\alpha_{1} D}$ & $\frac{\beta_{1}+\alpha_{1} \delta_{1}}{X+\alpha_{1} D}$ & $\frac{\beta_{2}+\alpha_{1} \delta_{2}}{X+\alpha_{1} D}$ \\
\hline
\end{tabular}

Table 5. The profile of equilibrium allocations where $\rho_{I I I} \equiv\left(1+\beta_{1}+\alpha_{1} \delta_{1}\right)\left(\delta_{1}+\alpha_{2} \beta_{1}\right) / K$

$$
\begin{gathered}
\rho_{I V} \equiv\left(1+\beta_{1}+\alpha_{1} \delta_{1}\right)\left(\delta_{2}+\alpha_{2} \beta_{2}\right) / L, \rho_{V} \equiv\left(\delta_{2}+\alpha_{2} \beta_{2}\right)\left[X+\alpha_{1}\left(\delta_{1}+\delta_{2}\right)\right] / L \\
K \equiv \delta_{1}+\alpha_{2} \beta_{1}+\left(\beta_{1}+\alpha_{1} \delta_{1}\right)\left[D+\alpha_{2}\left(\beta_{1}+\beta_{2}\right)\right] \\
\text { and } L \equiv \beta_{2}+\alpha_{1} \delta_{2}+\left(\delta_{2}+\alpha_{2} \beta_{2}\right)\left[X+\alpha_{1}\left(\delta_{1}+\delta_{2}\right)\right]
\end{gathered}
$$

Figure 7 illustrates the pattern of household demands for the respective public goods under non-identical Cobb-Douglas utility functions with altruistic preferences. We can draw a graph 


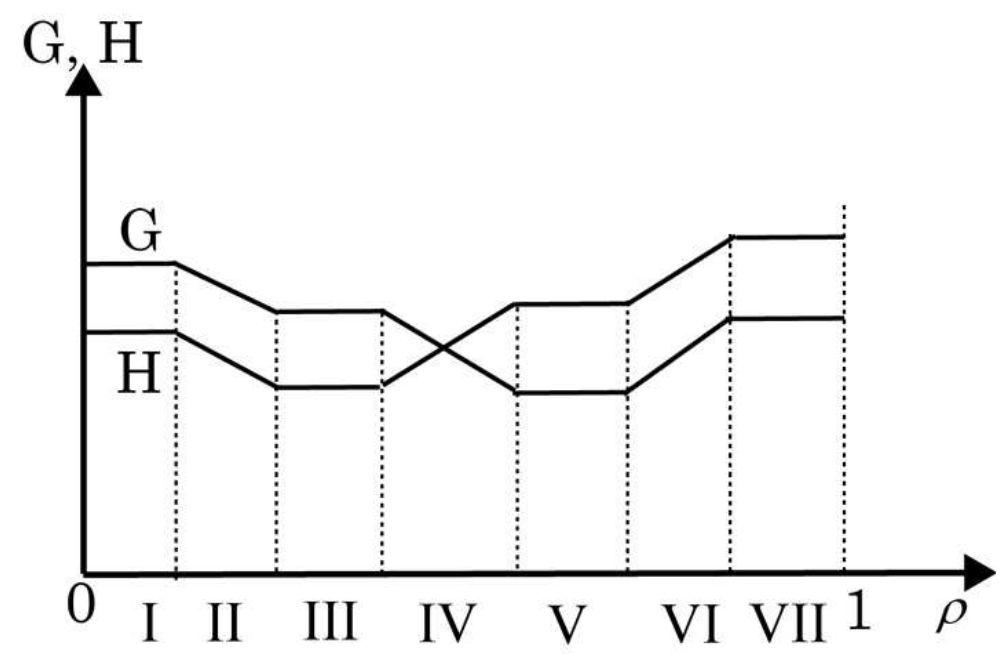

Figure 7: The aggregate quantities of the two public goods under two-sided caring preferences for the social welfare function with flat segments at its extremes as well as with two inverse $U$-shaped portions. From these similarities, it may be concluded that the major difference between egoistic and altruistic preferences is that the latter preferences have flat segments when the distribution of income is extremely unequal.

\section{Concluding Remarks}

Although several papers have formally incorporated the possibility of many types of public goods, little attention has been paid to the welfare implications of such models in the literature on voluntary provision of public goods. To fill this gap, we have shown how the presence of multiple public goods as well as altruistic preferences introduces a new aspect to the consideration of the welfare implications of the two types of redistribution policy, namely, inequality-raising and income-equalizing redistribution policies. This paper has shown that not only income-inequality raising but also income-equalizing redistribution policies may raise social welfare in the presence of multiple voluntarily supplied pure public goods, which sharply contradicts the conclusion of Itaya et al. (1997).

However, we present two caveats. First, the income-equalizing policy is effective such that it may raise social welfare only when individual preferences are non-identical, whereas when individual preferences are identical, the income-equalizing redistribution is no longer effective 
and thus leaves social welfare invariant in terms of distribution of income. Thus, the distinction between identical and non-identical preferences is critical in evaluating the welfare analysis of a redistribution policy, especially when the incomes of individuals do not differ very much. Second, whenever altruistically motivated voluntary transfers are operative, the inequalityraising redistribution policy is no longer effective; thus, in this case, the government need not engage in any redistribution policy.

This paper presents several important policy implications. First, the desirability of an income inequality-raising redistribution policy would be robust regardless of the number of voluntarily provided public goods unless altruistic motives are operative. Nevertheless, from our findings of this paper, we should not give too much credit to the policy prescription suggested by Itaya et al. (1997), in that it is always optimal to create a rich individual who becomes the sole provider of public goods. Moreover, Atkinson (1983) has justified the complete equality of income by adopting highly simplified assumptions to construct the social welfare function, such as (i) equal marginal social welfare weight, (ii) everyone has the same preferences, and (iii) diminishing marginal utility of income. Many economists following Atkinson have adopted more general social welfare functions with varying marginal social welfare weights, but they maintain the assumption of identical preferences. However, as demonstrated in this paper, the assumption of identical preferences is no longer a harmless assumption in the presence of multiple voluntarily supplied public goods. We should bear in mind that the assumption of identical preferences has very strong implications for the optimal income distribution policy, which could lead to inappropriate implications in issues of income distribution in actual economies where multiple pure public goods are voluntarily supplied.

Certainly, more research is needed to better understand the relationship between multiple pure public goods and social welfare. First, the most natural extension is to extend the present model to explicitly include more than two public goods or more than two persons. This extension would provide richer welfare implications. In particular, a more thorough analysis is called for to assess a model with many heterogeneous agents. Second, it would also be interesting to introduce distortionary taxes in the present setting of multiple public goods. More interestingly, we may analyze optimal distortionary taxes such as income and 
commodity taxes, which aim at the first-best or the second-best allocation. The analysis of this paper is a first step toward addressing more interesting and richer issues in a multiple public good setting.

\section{Appendix A: Comparative Statics}

We take the differentials of the system consisting of (4) and (5), to obtain

$$
\begin{gathered}
{\left[u_{G G}^{1}+u_{x x}^{1}-2 u_{x G}^{1}\right] d g_{1}+\left[u_{G H}^{1}-u_{x H}^{1}\right] d h_{2}=\left[-u_{G x}^{1}+u_{x x}^{1}\right] d \rho} \\
{\left[u_{H G}^{2}-u_{x G}^{2}\right] d g_{1}+\left[u_{H H}^{2}+u_{x x}^{2}-2 u_{x H}^{2}\right] d h_{2}=\left[-u_{H x}^{2}+u_{x x}^{2}\right] d(1-\rho) .}
\end{gathered}
$$

Applying Cramer's formula to (A.1) yields

$$
\begin{aligned}
\frac{d g_{1}}{d \rho} & =\underbrace{H^{-1}}_{+} \underbrace{\left(-u_{G x}^{1}+u_{x x}^{1}\right)}_{-} \underbrace{\left(u_{H H}^{2}+u_{x x}^{2}-2 u_{x H}^{2}\right)}_{-}>0, \\
\frac{d g_{1}}{d(1-\rho)} & =-\underbrace{H^{-1}}_{+} \underbrace{\left(-u_{H x}^{2}+u_{x x}^{2}\right)}_{-} \underbrace{\left(u_{G H}^{1}-u_{x H}^{1}\right)}_{-}<0, \\
\frac{d h_{2}}{d \rho} & =-\underbrace{H^{-1}}_{+} \underbrace{\left(-u_{G x}^{1}+u_{x x}^{1}\right)}_{-} \underbrace{\left(u_{H G}^{2}-u_{x G}^{2}\right)}_{-}<0, \\
\frac{d h_{2}}{d(1-\rho)} & =\underbrace{H^{-1}}_{+} \underbrace{\left(u_{G G}^{1}+u_{x x}^{1}-2 u_{x G}^{1}\right)}_{-} \underbrace{\left(-u_{H x}^{2}+u_{x x}^{2}\right)}_{-}>0,,
\end{aligned}
$$

where

$$
H \equiv\left|\begin{array}{cc}
u_{G G}^{1}+u_{x x}^{1}-2 u_{x G}^{1} & u_{G H}^{1}-u_{x H}^{1} \\
u_{H G}^{2}-u_{x G}^{2} & u_{H H}^{2}+u_{x x}^{2}-2 u_{x H}^{2}
\end{array}\right|>0 .
$$

The signs of (A.2)-(A.5) can be found from (A.6), (A.9), (A.10), (A.11), and (A.12). First, the sign of the determinant $H$ in (A.6) is positive from the assumed stability condition of the Nash equilibrium under the best-reply dynamics process. To ensure stability for the Nash equilibrium corresponding to Case 7 , the relative slope of the best-reply functions of both 
individuals must satisfy the following condition: ${ }^{13}$

$$
\left|\frac{d h_{2}}{d g_{1}}\right|_{1^{\prime} \text { s reaction }}>\left|\frac{d h_{2}}{d g_{1}}\right|_{2^{\prime} \text { s reaction }},
$$

where the best-reply functions of individuals 1 and 2 are given by $g_{1}=g_{1}\left(h_{2}\right)$ and $h_{2}=h_{2}\left(g_{1}\right)$, which are derived from (4) and (5), respectively. The slopes of the respective best-reply functions are thus given by

$$
\begin{aligned}
& \left.\frac{d h_{2}}{d g_{1}}\right|_{1^{\prime} \text { s reaction }}=-\frac{u_{G G}^{1}+u_{x x}^{1}-2 u_{x G}^{1}}{u_{G H}^{1}-u_{x H}^{1}}<0, \\
& \left.\frac{d h_{2}}{d g_{1}}\right|_{2^{\prime} \text { s reaction }}=-\frac{u_{H G}^{2}-u_{x G}^{2}}{u_{H H}^{2}+u_{x x}^{2}-2 u_{x H}^{2}}<0 .
\end{aligned}
$$

which, together with (A.7), imply that

$$
\left|-\frac{u_{G G}^{1}+u_{x x}^{1}-2 u_{x G}^{1}}{u_{G H}^{1}-u_{x H}^{1}}\right|_{1^{\prime} \text { s reaction }}>\left|-\frac{u_{H G}^{2}-u_{x G}^{2}}{u_{H H}^{2}+u_{x x}^{2}-2 u_{x H}^{2}}\right|_{2^{\prime} \text { s reaction }},
$$

or, equivalently,

$$
\frac{u_{G G}^{1}+u_{x x}^{1}-2 u_{x G}^{1}}{u_{G H}^{1}-u_{x H}^{1}}>\frac{u_{H G}^{2}-u_{x G}^{2}}{u_{H H}^{2}+u_{x x}^{2}-2 u_{x H}^{2}} .
$$

Since $u_{G G}^{1}+u_{x x}^{1}-2 u_{x G}^{1}<0$ and $u_{H H}^{2}+u_{x x}^{2}-2 u_{x H}^{2}<0$ from the assumption of strict quasiconcavity, and since both sides of (A.8) are positive, it turns out that

$$
\begin{gathered}
u_{G H}^{1}-u_{x H}^{1}<0 \text { and } u_{H G}^{2}-u_{x G}^{2}<0, \\
\left(u_{H H}^{1}+u_{x x}^{1}-2 u_{x H}^{1}\right)\left(u_{G G}^{2}+u_{x x}^{2}-2 u_{x G}^{2}\right)>\left(u_{G H}^{2}-u_{x H}^{2}\right)\left(u_{H G}^{1}-u_{x G}^{1}\right),
\end{gathered}
$$

the latter of which implies that the sign of $H$ in (A.8) is positive.

Similarly, we can compute the respective slopes of the best-reply functions $h_{1}=h_{1}\left(g_{2}\right)$ and $g_{2}=g_{2}\left(h_{1}\right)$ given by $(9)$ and $(10)$ :

$$
\begin{aligned}
& \left.\frac{d g_{2}}{d h_{1}}\right|_{1^{\prime} \text { s reaction }}=-\frac{u_{H H}^{1}+u_{x x}^{1}-2 u_{x H}^{1}}{u_{H G}^{1}-u_{x G}^{1}}<0, \\
& \left.\frac{d g_{2}}{d h_{1}}\right|_{2^{\prime} \text { s reaction }}=-\frac{u_{G H}^{2}-u_{x H}^{2}}{u_{G G}^{2}+u_{x x}^{2}-2 u_{x G}^{2}}<0 .
\end{aligned}
$$

\footnotetext{
${ }^{13}$ There are several variations in formulating an adjustment process to reach a Nash equilibrium. Among them, we focus on the continuous-time version of best-response dynamics. Contributor $i=1,2$ has a welldefined best-response function, $g_{1}=g_{1}\left(h_{2}\right)$ and $h_{2}=h_{2}\left(g_{1}\right)$, respectively, and the dynamics can thus be written as

$$
\left[\begin{array}{l}
\dot{g}_{1} \\
\dot{h}_{2}
\end{array}\right]=\kappa\left[\begin{array}{l}
g_{1}\left(h_{2}\right)-g_{1} \\
h_{2}\left(g_{1}\right)-h_{2}
\end{array}\right],
$$

where $\kappa$ is a vector of positive constants representing an adjustment speed. Asymptotic local stability of an equilibrium requires that, in a neighborhood of the rest point (i.e., a Nash equilibrium), the product of each individual's response to a change in the other's contribution should be negative. In a two-person contribution game, this condition is equivalent to the negative sign of the Jacobian of the linearized system of the above dynamic system.
} 
The stability condition for this Nash equilibrium under Case 8 gives

$$
\frac{u_{H H}^{1}+u_{x x}^{1}-2 u_{x H}^{1}}{u_{H G}^{1}-u_{x G}^{1}}>\frac{u_{G H}^{2}-u_{x H}^{2}}{u_{G G}^{2}+u_{x x}^{2}-2 u_{x G}^{2}}>0,
$$

which, together with the assumption of strict quasi-concavity (i.e., $u_{H H}^{1}+u_{x x}^{1}-2 u_{x H}^{1}<0$ and $\left.u_{G G}^{2}+u_{x x}^{2}-2 u_{x G}^{2}<0\right)$, yields

$$
u_{H G}^{1}-u_{x G}^{1}<0 \text { and } u_{G H}^{2}-u_{x H}^{2}<0 .
$$

The normality assumption for every good, in contrast, results in

$$
\begin{aligned}
& \frac{d x_{1}}{d \rho}>0 \text { if and only if }\left(u_{G G}^{1}-u_{x G}^{1}\right)\left(u_{H H}^{1}-u_{x H}^{1}\right)>\left(u_{G H}^{1}-u_{x H}^{1}\right)\left(u_{H G}^{1}-u_{x G}^{1}\right), \\
& \frac{d g_{2}}{d \rho}>0 \text { if and only if }\left(u_{G x}^{2}-u_{x x}^{2}\right)\left(u_{H H}^{2}-u_{x H}^{2}\right)<\left(u_{G H}^{2}-u_{x H}^{2}\right)\left(u_{H x}^{2}-u_{x x}^{2}\right), \\
& \frac{d h_{1}}{d \rho}>0 \text { if and only if }\left(u_{G x}^{1}-u_{x x}^{1}\right)\left(u_{H G}^{1}-u_{x G}^{1}\right)>\left(u_{G G}^{1}-u_{x G}^{1}\right)\left(u_{H x}^{1}-u_{x x}^{1}\right) .
\end{aligned}
$$

Combining these normality conditions with (A.9) and (A.11) confirms that ${ }^{14}$

$$
-u_{G x}^{1}+u_{x x}^{1}<0 \text { and }-u_{H x}^{2}+u_{x x}^{2}<0 .
$$

\section{Appendix B: Characterization of Caring Preferences}

(i) Suppose that Case 5 (i.e., $g_{1}=0, h_{1}=0, g_{2}>0$, and $h_{2}>0$ ) is consistent with $t_{1}>0$ and $t_{2}=0$. The first-order conditions for this profile are given by the following conditions:

$$
\begin{gathered}
-u_{x}^{1}+u_{G}^{1}+\alpha_{1} u_{G}^{2}<0 \text { and }-u_{x}^{1}+u_{H}^{1}+\alpha_{1} u_{H}^{2}<0, \\
-u_{x}^{2}+u_{G}^{2}+\alpha_{2} u_{G}^{1}=0 \text { and }-u_{x}^{2}+u_{H}^{2}+\alpha_{2} u_{H}^{1}=0, \\
-u_{x}^{1}+\alpha_{1} u_{x}^{2}=0 \text { and }-u_{x}^{2}+\alpha_{2} u_{x}^{1}<0,
\end{gathered}
$$

where $u^{1} \equiv u^{1}\left(\rho-t_{1}, g_{2}, h_{2}\right)$ and $u^{2} \equiv u^{2}\left(1-\rho-g_{2}-h_{2}+t_{1}, g_{2}, h_{2}\right)$.

Combining these conditions yields

$$
\begin{aligned}
u_{x}^{1} & =\alpha_{1} u_{x}^{2}=\alpha_{1}\left(u_{G}^{2}+\alpha_{2} u_{G}^{1}\right)=\alpha_{1} u_{G}^{2}+\alpha_{2} \alpha_{1} u_{G}^{1} \\
& <u_{x}^{1}-u_{G}^{1}+\alpha_{2} \alpha_{1} u_{G}^{1}=u_{x}^{1}+\left(\alpha_{2} \alpha_{1}-1\right) u_{G}^{1},
\end{aligned}
$$

thus implying that $0<\left(\alpha_{2} \alpha_{1}-1\right) u_{G}^{1}$, contradicting the assumption that $\alpha_{2} \alpha_{1}<1$.

When $t_{1}=0$ and $t_{2}>0$, in contrast, the only last two conditions in (A.13) are modified as follows:

$$
-u_{x}^{1}+\alpha_{1} u_{x}^{2}<0 \text { and }-u_{x}^{2}+\alpha_{2} u_{x}^{1}=0,
$$

where $u^{1} \equiv u^{1}\left(\rho+t_{2}, g_{2}, h_{2}\right)$ and $u^{2} \equiv u^{2}\left(1-\rho-g_{2}-h_{2}-t_{2}, g_{2}, h_{2}\right)$. In this case,

$$
\begin{aligned}
u_{x}^{2} & =\alpha_{2} u_{x}^{1}>\alpha_{2}\left(u_{G}^{1}+\alpha_{1} u_{G}^{2}\right)=\alpha_{2} u_{G}^{1}+\alpha_{2} \alpha_{1} u_{G}^{2} \\
& =u_{x}^{2}-u_{G}^{2}+\alpha_{2} \alpha_{1} u_{G}^{2}=u_{x}^{2}+\left(\alpha_{2} \alpha_{1}-1\right) u_{G}^{2},
\end{aligned}
$$

\footnotetext{
${ }^{14}$ Detailed derivations are available from the corresponding author upon request.
} 
thus implying that $\left(\alpha_{2} \alpha_{1}-1\right) u_{G}^{2}<0$, which is consistent with the assumption that $\alpha_{2} \alpha_{1}<1$.

(ii) We now consider Case 6 (i.e., $g_{1}>0, h_{1}>0, g_{2}=0$, and $h_{2}=0$ ) along with $t_{1}=0$ and $t_{2}>0$. The first-order conditions for this profile are given by the following conditions:

$$
\begin{gathered}
-u_{x}^{1}+u_{G}^{1}+\alpha_{1} u_{G}^{2}=0 \text { and }-u_{x}^{1}+u_{H}^{1}+\alpha_{1} u_{H}^{2}=0, \\
-u_{x}^{2}+u_{G}^{2}+\alpha_{2} u_{G}^{1}<0 \text { and }-u_{x}^{2}+u_{H}^{2}+\alpha_{2} u_{H}^{1}<0, \\
-u_{x}^{1}+\alpha_{1} u_{x}^{2}<0 \text { and }-u_{x}^{2}+\alpha_{2} u_{x}^{1}=0,
\end{gathered}
$$

where $u^{1} \equiv u^{1}\left(\rho-g_{1}-h_{1}+t_{2}, g_{1}, h_{1}\right)$ and $u^{2} \equiv u^{2}\left(1-\rho-t_{2}, g_{1}, h_{1}\right)$.

Combining these conditions yields

$$
\begin{aligned}
u_{x}^{2} & =\alpha_{2} u_{x}^{1}>\alpha_{2}\left(u_{G}^{1}+\alpha_{1} u_{G}^{2}\right)=\alpha_{2} u_{G}^{1}+\alpha_{2} \alpha_{1} u_{G}^{2} \\
& =u_{x}^{2}-u_{G}^{2}+\alpha_{2} \alpha_{1} u_{G}^{2}=u_{x}^{2}+\left(\alpha_{2} \alpha_{1}-1\right) u_{G}^{2},
\end{aligned}
$$

which leads to $\left(\alpha_{2} \alpha_{1}-1\right) u_{G}^{2}<0$, consistent with the assumption that $\alpha_{2} \alpha_{1}<1$.

Following the first part of the proof of (i), we can prove that Case 6 (i.e., $g_{1}>0, h_{1}>0$, $g_{2}=0$, and $\left.h_{2}=0\right)$ along with $t_{1}=0$ and $t_{2}>0$ does not occur.

(iii) Suppose that Case 7 (i.e., $g_{1}>0, h_{1}=0, g_{2}=0$, and $h_{2}>0$ ) is consistent with $t_{1}>0$ and $t_{2}=0$, that is,

$$
\begin{gathered}
-u_{x}^{1}+u_{G}^{1}+\alpha_{1} u_{G}^{2}=0 \text { and }-u_{x}^{1}+u_{H}^{1}+\alpha_{1} u_{H}^{2}<0, \\
-u_{x}^{2}+u_{G}^{2}+\alpha_{2} u_{G}^{1}<0 \text { and }-u_{x}^{2}+u_{H}^{2}+\alpha_{2} u_{H}^{1}=0, \\
-u_{x}^{1}+\alpha_{1} u_{x}^{2}=0 \text { and }-u_{x}^{2}+\alpha_{2} u_{x}^{1}<0,
\end{gathered}
$$

where $u^{1} \equiv u^{1}\left(\rho-g_{1}-t_{1}, g_{1}, h_{2}\right)$ and $u^{2} \equiv u^{2}\left(1-\rho-h_{2}+t_{1}, g_{1}, h_{2}\right)$. Combining these conditions leads to

$$
\begin{aligned}
u_{x}^{1} & =\alpha_{1} u_{x}^{2}=\alpha_{1}\left(u_{H}^{2}+\alpha_{2} u_{H}^{2}\right)=\alpha_{1} u_{H}^{2}+\alpha_{1} \alpha_{2} u_{H}^{2} \\
& <u_{x}^{1}-u_{H}^{1}+\alpha_{1} \alpha_{2} u_{H}^{2}=u_{x}^{2}+\left(\alpha_{1} \alpha_{2}-1\right) u_{G}^{2},
\end{aligned}
$$

thus yielding $\left(\alpha_{1} \alpha_{2}-1\right) u_{G}^{2}>0$, which is a contradiction.

Next, suppose that Case 7 is consistent with $t_{1}=0$ and $t_{2}>0$, that is,

$$
-u_{x}^{1}+\alpha_{1} u_{x}^{2}<0 \text { and }-u_{x}^{2}+\alpha_{2} u_{x}^{1}=0,
$$

where $u^{1} \equiv u^{1}\left(\rho-g_{1}+t_{2}, g_{1}, h_{2}\right)$ and $u^{2} \equiv u^{2}\left(1-\rho-h_{2}-t_{2}, g_{1}, h_{2}\right)$. Combining these conditions yields

$$
\begin{aligned}
u_{x}^{2} & =\alpha_{2} u_{x}^{1}=\alpha_{2}\left(u_{G}^{1}+\alpha_{1} u_{G}^{2}\right)=\alpha_{2} u_{G}^{1}+\alpha_{2} \alpha_{1} u_{G}^{2} \\
& <u_{x}^{2}-u_{G}^{2}+\alpha_{2} \alpha_{1} u_{G}^{2}=u_{x}^{2}+\left(\alpha_{2} \alpha_{1}-1\right) u_{G}^{2},
\end{aligned}
$$

thus yielding $\left(\alpha_{2} \alpha_{1}-1\right) u_{G}^{2}>0$, which is a contradiction. To sum up, in Case 7, no voluntary income transfers takes place between individuals.

Following the same procedure used for Case 7 , we can prove that no voluntary income transfers takes place between individuals in Case 8 . 


\section{Appendix C: Derivations for Figures 2, 4, and 6}

(i) In Subsection 4.1, the utilitarian social welfare function composed of egoistic and identical Cobb-Douglas utility functions is given by

$$
W=\log x_{1}+\log x_{2}+2 \beta_{1} \log G+2 \beta_{2} \log H .
$$

First, note that since the levels of each individual's private consumption and the two public goods in Regime $I I$ remain invariant with respect to alternations in the distribution of income, so does the level of social welfare. In contrast, since in Regime $I$ (i.e., $g_{1}=h_{1}=0, g_{2}>0$, $h_{2}>0$ ), the social welfare function is given by

$$
W^{I} \equiv \log \rho+\log \frac{1-\rho}{X}+2 \beta_{1} \log \frac{\beta_{1}(1-\rho)}{X}+2 \beta_{2} \log \frac{\beta_{2}(1-\rho)}{X},
$$

differentiating $W^{I}$ with respect to $\rho$ yields

$$
\frac{d W^{I}}{d \rho}=\frac{1}{\rho}-\frac{1+2 \beta_{1}+2 \beta_{2}}{1-\rho} \gtreqless 0 \quad \text { if and only if } \rho \lesseqgtr \frac{1}{2 X},{ }^{15}
$$

which implies that function $W^{I}$ achieves a peak at $\rho=1 /(2 X)$, while $W^{I}$ increases (decreases) with $\rho$ so long as $\rho<1 /(2 X)(\rho>1 /(2 X))$. Similarly, differentiating the welfare function defined over Regime $I I I$ (i.e., $g_{1}>0, h_{1}>0, g_{2}=h_{2}=0$ ),

$$
W^{I I I} \equiv \log \frac{\rho}{X}+\log (1-\rho)+2 \beta_{1} \log \frac{\beta_{1} \rho}{X}+2 \beta_{2} \log \frac{\beta_{2} \rho}{X},
$$

with respect to $\rho$ yields

$$
\frac{d W^{I I I}}{d \rho}=\frac{1+2 \beta_{1}+2 \beta_{2}}{\rho}-\frac{1}{1-\rho} \gtreqless 0 \quad \text { if and only if } \rho \lesseqgtr \frac{\beta_{1}+\beta_{2}+X}{X} .{ }^{16}
$$

(ii) In Subsection 4.2, the social welfare function composed of altruistic and identical Cobb-Douglas utility functions is given by

$$
W=(1+\alpha) \log x_{1}+(1+\alpha) \log x_{2}+2 \beta_{1}(1+\alpha) \log G+2 \beta_{2}(1+\alpha) \log H .
$$

Note that the levels of social welfare in Regimes $I, I I I$, and $V$ remain constant in terms of the distribution of income. Substituting the corresponding values listed in Regime $I I$ (i.e., $g_{1}=h_{1}=0, g_{2}>0, h_{2}>0, t_{1}=t_{2}=0$ ) of Table 3 into (28) yields

$$
\begin{aligned}
W^{I I} & \equiv(1+\alpha) \log \rho+(1+\alpha) \log \frac{1-\rho}{\alpha\left(\beta_{1}+\beta_{2}\right)+X} \\
& +2 \beta_{1}(1+\alpha) \log \frac{\beta_{1}(1+\alpha)(1-\rho)}{\alpha\left(\beta_{1}+\beta_{2}\right)+X}+2 \beta_{2}(1+\alpha) \log \frac{\beta_{2}(1+\alpha)(1-\rho)}{\alpha\left(\beta_{1}+\beta_{2}\right)+X}
\end{aligned}
$$

\footnotetext{
${ }^{15}$ The fact that $\frac{1}{1+X}-\frac{1}{2 X}=\frac{\beta_{1}+\beta_{2}}{2 X(1+X)}>0$ implies that the peak (i.e., a local maximum) of the function $W^{I}$ is situated in the range of income distribution $\rho \in[0,1 /(1+X)]$, as illustrated in Figure 2 .

${ }^{16}$ Since $\frac{\beta_{1}+\beta_{2}+X}{X}-\frac{X}{1+X}=\frac{\beta_{1}+\beta_{2}}{2 X(1+X)}>0$, the peak of the function $W^{I I I}$ is situated in the range of income distribution $\rho \in[X /(1+X), 1)$, as illustrated in Figure 2 .
} 
which gives ${ }^{17}$

$$
\frac{d W^{I I}}{d \rho}=(1+\alpha)\left(\frac{1}{\rho}-\frac{1+2 \beta_{1}+2 \beta_{2}}{1-\rho}\right) \gtreqless 0 \text { if and only if } \rho \lesseqgtr \frac{1}{2 X} .
$$

In Regime $I V$ (i.e., $g_{1}>0, h_{1}>0, g_{2}=h_{2}=0, t_{1}=t_{2}=0$ ), differentiating the social welfare function

$$
\begin{aligned}
W^{I V} & \equiv(1+\alpha) \log \frac{\rho}{\alpha\left(\beta_{1}+\beta_{2}\right)+X}+(1+\alpha) \log 1-\rho \\
& +2 \beta_{1}(1+\alpha) \log \frac{\beta_{1}(1+\alpha) \rho}{\alpha\left(\beta_{1}+\beta_{2}\right)+X}+2 \beta_{2}(1+\alpha) \log \frac{\beta_{2}(1+\alpha) \rho}{\alpha\left(\beta_{1}+\beta_{2}\right)+X}
\end{aligned}
$$

with respect to $\rho$ yields ${ }^{18}$

$$
\frac{d W^{I V}}{d \rho}=(1+\alpha)\left(\frac{1+2 \beta_{1}+2 \beta_{2}}{\rho}-\frac{1}{1-\rho}\right) \gtreqless 0 \text { if and only if } \rho \lesseqgtr \frac{\beta_{1}+\beta_{2}+X}{2 X} .
$$

(iii) In Subsection 5.1, the social welfare function composed of egoistic and non-identical Cobb-Douglas utility functions is given by

$$
W=\log x_{1}+\log x_{2}+\left(\beta_{1}+\delta_{1}\right) \log G+\left(\beta_{2}+\delta_{2}\right) \log H .
$$

Note that the levels of social welfare in Regimes $I I$ and $I V$ remain invariant with respect to the distribution of income. In contrast, since the social welfare function defined over Regime $I$ (i.e., $g_{1}=h_{1}=0, g_{2}>0, h_{2}>0$ ) is given by

$$
W^{I} \equiv \log \rho+\log \frac{1-\rho}{D}+\left(\beta_{1}+\delta_{1}\right) \log \frac{\delta_{1}(1-\rho)}{D}+\left(\beta_{2}+\delta_{2}\right) \log \frac{\delta_{2}(1-\rho)}{D},
$$

differentiating $W^{I}$ with respect to $\rho$ yields ${ }^{19}$

$$
\frac{d W^{I}}{d \rho}=\frac{1}{\rho}-\frac{1+\beta_{1}+\delta_{1}+\beta_{2}+\delta_{2}}{1-\rho} \gtreqless 0 \text { if } \rho \lesseqgtr \frac{1}{D+X} .
$$

Since the shape of the social welfare function defined over Regime $I I I$ (i.e., $g_{1}>0, h_{1}=0$, $\left.g_{2}=0, h_{2}>0\right)$ has been derived in the text, it is omitted here. Finally, differentiating the social welfare function defined over Regime $V$ (i.e., $g_{1}>0, h_{1}>0, g_{2}=h_{2}=0$ ),

$$
W^{V} \equiv \log \frac{\rho}{X}+\log 1-\rho+\left(\beta_{1}+\delta_{1}\right) \log \frac{\beta_{1} \rho}{X}+\left(\beta_{2}+\delta_{2}\right) \log \frac{\beta_{2} \rho}{X},
$$

\footnotetext{
${ }^{17}$ Since $\frac{1}{2 X}-\frac{\alpha}{(1+\alpha) X}=\frac{1}{X} \frac{1-\alpha}{2(2+\alpha)}>0$, and $\frac{1}{1+\alpha\left(\beta_{1}+\beta_{2}\right)+X}-\frac{1}{2 X}=\frac{(1-\alpha)\left(\beta_{1}+\beta_{2}\right)}{2 X\left[1+\alpha\left(\beta_{1}+\beta_{2}\right)+X\right]}>0$, the peak of the function $W^{I I}$ is located in Region $I I$ of Figure 4.

${ }^{18}$ Since $\frac{\beta_{1}+\beta_{2}+X}{2 X}-\frac{\alpha\left(\beta_{1}+\beta_{2}\right)+X}{1+\alpha\left(\beta_{1}+\beta_{2}\right)+X}=\frac{\left(\beta_{1}+\beta_{2}\right)(1-\alpha+2 X)}{2 X\left(1+\alpha\left(\beta_{1}+\beta_{2}\right)+X\right)}>0$, and $\frac{\alpha\left(\beta_{1}+\beta_{2}\right)+X}{(1+\alpha) X}-\frac{\beta_{1}+\beta_{2}+X}{2 X}=\frac{1-\alpha}{2 X(1+\alpha)}>0$, the peak of the function $W^{I V}$ is located in Region $I V$ of Figure 4.

${ }^{19}$ Since $\frac{\delta_{1}}{\delta_{1}+\beta_{1} D}-\frac{1}{D+X}=\frac{\delta_{1}+\left(2 \delta_{1}-\beta_{1}\right)\left(\beta_{1}+\beta_{2}\right)}{\left(\delta_{1}+\beta_{1} D\right)(D+X)}>0$ due to Assumption 4, the peak of the function $W^{I}$ is situated in Region $I$ of Figure 6.
} 
with respect to $\rho$ yields ${ }^{20}$

$$
\frac{d W^{V}}{d \rho}=\frac{X+\delta_{1}+\delta_{2}-\rho(D+X)}{\rho(1-\rho)} \gtreqless 0 \text { if } \rho \lesseqgtr \frac{X+\delta_{1}+\delta_{2}}{D+X} .
$$

\section{References}

Atkinson, A. (1983) The Economics of Inequality, second ed., Oxford University Press, New York. sect. 3.4, 53-59.

Bergstrom, T., L. Blume, and H. Varian (1986) On the private provision of public goods, Journal of Public Economics 29, 25-49.

Browning, M., P.-A., Chiappori, and Y. Weiss (2014) Economics of the Family (Cambridge Surveys of Economic Literature).

Cornes R., and T. Sandler (2000) Pareto improving redistribution and pure public goods, German Economic Review 1, 169-186.

Cornes, R., and J. Itaya (2010) On the private provision of two or more public goods, Journal of Public Economic Theory 12, 363-385.

Cornes, R., J. Itaya, and A. Tanaka (2012) Private provision to public goods among families, Journal of Population Economics 12, 1451-1480.

Cornes, R. C. and Schweinberger, A. S. (1996) Free riding and the inefficiency of the private production of pure public goods, Canadian Journal of Economics 29, 70-91.

Dasgupta, I. (2009) Why praise inequality? Public good provision, income distribution and social welfare, mimeo.

Ghosh, S., Karaivanov, A. and M. Oak (2007) A case for bundling public goods contribution, Journal of Public Economic Theory 98, 425-450.

Kemp, M. C. (1984) A note on the theory of international transfers, Economics Letters 14, $2-3$.

Itaya, J., De Meza, and G.Myles (1997) In praise of inequality: public good provision and income distribution, Economic Letters 57, 289-296.

Mukherjee, V., and T. Sanyal (2011) A note on welfare effect and desirability of inequalityraising transfers, Annals for Public and Cooperative Economics 57, 301-311.

OECD (2012) OECD Economic Surveys, Norway, OECD.

Warr, P. (1983) The private provision of a public good is independent of the distribution of income, Economics Letters 13, 207-211.

\footnotetext{
${ }^{20}$ Since $\frac{\delta_{1}+\delta_{2}+X}{D+X}-\frac{\delta_{2} X}{\beta_{2}+\delta_{2} X}=\frac{\left(\beta_{2}-\delta_{2}\right)\left(1+\beta_{1}+\beta_{2}\right)+\beta_{2}\left(\delta_{1}+\delta_{2}\right)}{(D+X)\left(\beta_{2}+\delta_{2} X\right)}>0$ due to Assumption 4, the peak of the social welfare function is situated in Region $V$ of Figure 6.
} 